\title{
Present and projected sensitivities of Dark Matter direct detection experiments to effective WIMP-nucleus couplings
}

\author{
Sunghyun Kang, Stefano Scopel, Gaurav Tomar, Jong-Hyun Yoon \\ Department of Physics, Sogang University, Seoul, Korea, 121-742
}

\begin{abstract}
Assuming for Weakly Interacting Massive Particles (WIMPs) a Maxwellian velocity distribution in the Galaxy we explore in a systematic way the relative sensitivity of an extensive set of existing and projected Dark Matter (DM) direct detection experiments to each of the 14 couplings that parameterize the most general non-relativistic (NR) effective Hamiltonian allowed by Galilean invariance for a contact interaction driving the elastic scattering off nuclei of WIMPs of spin $1 / 2$. We perform our analysis in terms of two free parameters: the WIMP mass $m_{\chi}$ and the ratio between the WIMP-neutron and the WIMP-proton couplings $c^{n} / c^{p}$. We include the modified signal spectral shape due to non-standard interactions when it is needed in the determination of the bound, such as in the case of background subtraction or of the application of the optimal-interval method. For each coupling, in the $m_{\chi}-c^{n} / c^{p}$ plane we provide contour plots of the most stringent 90\% C.L. bound on the WIMP-nucleon cross section and show the experiment providing it. We also introduce NRDD_constraints, a simple interpolating code written in Python that allows to obtain the numerical value of the bound as a function of the WIMP mass $m_{\chi}$ and of the coupling ratio $c^{n} / c^{p}$ for each NR coupling. We find that 9 experiments out of the 14 present Dark Matter searches considered in our analysis provide the most stringent bound on some of the effective couplings for a given choice of $\left(m_{\chi}, c^{n} / c^{p}\right)$ : this is evidence of the complementarity of different target nuclei and/or different combinations of count-rates and energy thresholds when the search of DM is extended to a wide range of possible interactions.
\end{abstract}

Keywords:

PACS: $95.35 .+\mathrm{d}$,

\section{Introduction}

Up to $27 \%$ of the total mass density of the Universe [1] and more than $90 \%$ of the halo of our Galaxy are believed to be composed of Dark Matter (DM). The properties of such invisible component are yet unknown since DM has been only observed through gravity so far. However, to comply with the Cold Dark Matter (CDM) paradigm of Galaxy formation and to be a viable thermal relic, in one of its most popular scenarios DM is believed to be composed of Weakly

Email addresses: francis735@naver.com (Sunghyun Kang), scopel@sogang.ac.kr (Stefano Scopel), tomar@sogang.ac.kr (Gaurav Tomar), jyoon@sogang.ac.kr (Jong-Hyun Yoon) 
Interacting Massive Particles (WIMPs) with a mass in the GeV-TeV range and weak-type interactions with ordinary matter. Such small but non vanishing interactions can drive WIMP scatterings off nuclear targets, and the measurement of the ensuing nuclear recoils in low-background detectors (direct detection) represents the most straightforward way to detect them. Indeed, a large worldwide effort is currently under way to observe WIMP-nuclear scatterings, but, with the exception of the DAMA collaboration [2, 3, 4, 5] that has been observing for a long time an excess compatible to the annual modulation of a DM signal, many other experiments using different nuclear targets and various background-subtraction techniques have failed to observe any WIMP signal so far [6, 7, 8, 9, 10, 11, 12, 13, 14, 15, 16, 17, 18].

The expected WIMP interaction scale happens to fit nicely to what is also believed to be the cut-off scale of the Standard Model (SM), beyond which new physics is expected to come on shell in order to stabilize the Higgs vacuum, and indeed most of the explicit ultraviolet completions of the SM contain WIMP exotic states that are viable DM candidates and for which detailed predictions for WIMP-nuclear scattering can be worked out. Crucially, this allows to determine how the WIMP interacts with different targets, and to compare in this way the sensitivity of different detectors to a given WIMP candidate, with the goal of choosing the most effective detection strategy.

A typical example of this approach is the direct search for the Supersymmetric neutralino, whose cross section off nuclei is usually driven either by a Higgs- or squark-exchange propagator, leading to a Spin Independent (SI) interaction that is the same for protons and neutrons (isoscalar) and scales with the square of the atomic mass number:

$$
\sigma_{\chi N} \propto\left[c^{p} Z+(A-Z) c^{n}\right]^{2},
$$

with $A$ the nuclear mass number, $Z$ the nuclear charge and $c^{p, n}$ the WIMP couplings to protons and neutrons, with $c^{n}=c^{p}$.

If indeed the neutralino-nucleus interaction amplitude is given by Eq.(1) the corresponding cross section is non-vanishing off any target and highly enhanced for heavy nuclei. Since expected signals are very low due to the very tight present constraints, very large exposures are required, as well as extremely low background levels that nowadays can only be achieved using discrimination techniques to distinguish nuclear recoils from natural radioactivity. This has naturally led to a very strong drive in the physics community to develop large-mass dual-phase (liquid and gaseous) xenon detectors [6, 19, 20] that indeed already provide today and are expected to provide in the future the most stringent bounds on this type of interaction.

Notice, however, that the current leading position of xenon detectors in the direct search of DM rests on the specific assumption of Eq. (1) for the scaling law of the WIMP-nucleon cross section with different targets. Two trivial counter-examples that show how there are viable DMnucleus interactions for which xenon might not be the optimal target to detect DM are provided by the case of isospin-violating models [21, 22] where the ratio between the WIMP-proton and the WIMP-neutron couplings is tuned to $c^{n} / c^{p} \simeq Z /(Z-A) \simeq-0.7$ to suppress the WIMP-xenon interaction amplitude, and by particles such as the Higgsino or a Majorana neutrino that couple to ordinary matter through a $Z$-boson propagator leading to a Spin-Dependent (SD) WIMPnucleon interaction:

$$
\mathcal{L}_{\text {int }} \ni c^{p} \vec{S}_{\chi} \cdot \vec{S}_{p}+c^{n} \vec{S}_{\chi} \cdot \vec{S}_{n}
$$

where $\vec{S}_{\chi}, \vec{S}_{n}$ and $\vec{S}_{p}$ are the spins of the WIMP, the neutron and the proton, respectively. 
In the case of an isospin-violating SI interaction, since the $A / Z$ ratio of all stable nuclear targets, including those used in direct detection, are not too far from unity, tuning $c^{n} / c^{p}$ to suppress the response of xenon inevitably leads also to a suppression of the WIMP scattering rates off all other targets. As a consequence, this would not only imply a different hierarchy among the sensitivity of different detector materials, but also an overall loss of sensitivity of present and future direct DM searches to the physics beyond the SM underlying DM. On the other hand, in the case of a spin-dependent interaction the relative sensitivity of different targets to the interaction $(\mathrm{Eq}, 2)$ is completely different from (Eq,1), since nucleon spins inside nuclei are not coherently enhanced. This implies that, at variance with the SI interaction, the SD one has no preference for heavy targets, so that the leading edge of xenon detectors compared to other targets is in general reduced. Moreover, isotopes with spin correspond to only about $47 \%$ of the overall target number in natural xenon, and since they have an even number of protons one has $\sum_{p} \vec{S}_{p} \rightarrow 0$ implying a strongly suppressed sensitivity to the $c^{p}$ coupling. As a consequence, the sensitivity of proton-odd targets such as those in fluorine detectors can be better than xenon when $c^{n} \ll c^{p}$.

In the present paper we wish to extend the discussion above, providing an assessment of the overall present and future sensitivity of direct detection experiments to WIMPs with the most general scaling law for WIMP-nucleus scattering, besides the SI and the SD one. In particular this task is achievable without fixing a specific high-energy extension of the SM since the WIMP-nucleus cross section can be parameterized in terms of the most general non-relativistic effective theory complying with Galilean symmetry, including a possible explicit dependence of the scattering cross section on the transferred momentum and of the WIMP incoming velocity [23, 24, 25, 26]. This approach, in which scales related to the spontaneous breaking of the chiral symmetry of QCD are integrated out, is alternative to incorporating the QCD constraints from chiral symmetry [27]. In our analysis, we will adopt for the velocity distribution $f(\vec{v})$ of the incoming WIMPs a standard thermalized non-relativistic gas described by a Maxwellian distribution. In particular, compared to other phenomenological analyses existing in the literature on WIMP-nucleus effective interactions [28, 29] in the present paper we wish to calculate updated constraints on the WIMP-nucleon cross section in the non-relativistic effective theory and provide a comparative discussion of the reach of different experiments to the various effective operators in order to show their complementarity in a transparent way.

Our approach will be relatively straightforward: we will consider an extensive list of present (XENON1T [6], PANDAX-II [7], KIMS [8], CDMSlite [9], SuperCDMS [10], COUPP [11], PICASSO [12], PICO-60 (using a $\mathrm{CF}_{3} \mathrm{I}$ target [13] and $\mathrm{C}_{3} \mathrm{~F}_{8}$ one [14]) CRESST-II [15. 30], DAMA (modulation data) [2, 3, 4, 5], DAMA0 (average count rate) [31]), CDEX [16] and DarkSide-50 (DS50) [18]) and future DM direct detection experiments (LUX-ZEPLIN (LZ) [20], PICO-500 [32] and COSINUS [33]) and the most general WIMP-nucleus effective Lagrangian for a WIMP particle of spin 1/2 scattering elastically off nuclei. Then, systematically assuming dominance of one of the possible interaction terms, we will provide for each of them a two-dimensional plot where the contours of the most stringent $90 \%$ C.L. upper bound 11 to an appropriately defined WIMP-nucleon effective cross section $\sigma_{\mathcal{N}}$ (that is related to the usual one in the case of interactions with a non-vanishing long-range asymptotic component such as the usual SI and SD cases) are shown as a function of the two parameters $m_{\chi}$ (WIMP mass), and $c^{n} / c^{p}$. Moreover, in the same $m_{\chi}-c^{n} / c^{p}$ plots, regions depicted with a different color will allow to determine which experiment provides the most stringent constraint for that particular choice

${ }^{1}$ For each experiment we apply a statistical treatment similar to that used for the published result, see Appendix B 
of parameters. To summarize our results for each coupling we will then provide as a function of the WIMP mass the maximal range spanned by the most constraining 90\% C.L. exclusion plot on the WIMP-nucleon cross section as a function of the WIMP mass when the ratio $c^{n} / c^{p}$ is varied. In Appendix D we will also introduce NRDD_constraints, a simple interpolating code written in Python that allows to obtain the numerical value of the most constraining limit on the effective cross section defined in Eq.(18) as a function of the WIMP mass $m_{\chi}$ and of the coupling ratio $c^{n} / c^{p}$ for each NR coupling.

In the present analysis we discuss one of the NR couplings at a time because they are the most general building blocks of the low-energy limit of any ultraviolet theory, so that an understanding of the behaviour of such couplings is crucial for the interpretation of more general scenarios containing the sum of several NR operator ${ }^{2}$ However, our results can be used also to estimate an approximate upper bound on the cross section in the case of the presence of more than one NR operator. The procedure to do so is discussed in Section 4 Our analysis is somewhat complementary to that of Ref. [34], where present WIMP direct detection experimental sensitivities are discussed for a limited number of non-relativistic operators and nuclear targets, but interferences among different operators are included in the discussion.

The paper is organized as follows. In Section 2 we summarize the non-relativistic Effective Field Theory (EFT) approach of Refs.[25, [26] and the formulas we use to calculate expected rates for WIMP-nucleus scattering; Section 3 is devoted to our quantitative analysis; in Section 4 we show how our results can be applied to the case of more than one NR operator. We will provide our conclusions in Section 5. In Appendix A we provide for completeness the WIMP response functions for the non-relativistic effective theory while in Appendix B we provide the details of each experiment included in the analysis. Appendix Cdescribes our treatment of the nuclear response functions for those isotopes for which a full calculation is not available in the literature. Finally, in Appendix D we introduce NRDD_constraints, a simple interpolating code written in Python that allows to retrieve the numerical value of the limits on the effective WIMP-nucleon cross section discussed in Section 3 and whose contour plots are shown in Figs. 3

\section{Summary of WIMP rates in non-relativistic effective models}

Making use of the non-relativistic EFT approach of Refs. [25, 26] the most general Hamiltonian density describing the WIMP-nucleus interaction can be written as:

$$
\mathcal{H}(\mathbf{r})=\sum_{\tau=0,1} \sum_{j=1}^{15} c_{j}^{\tau} O_{j}(\mathbf{r}) t^{\tau}
$$

where:

$$
O_{1}=1_{\chi} 1_{N} ; \quad O_{2}=\left(v^{\perp}\right)^{2} ; \quad O_{3}=i \vec{S}_{N} \cdot\left(\frac{\vec{q}}{m_{N}} \times \vec{v}^{\perp}\right) ;
$$

\footnotetext{
${ }^{2}$ Nevertheless, it is always possible to conceive a linear combination of relativistic operators leading to a single NR operator, since the number of the former is larger than that of the latter, although this might require a tuning of the couplings.
} 


$$
\begin{aligned}
O_{4} & =\vec{S}_{\chi} \cdot \vec{S}_{N} ; \quad O_{5}=i \vec{S}_{\chi} \cdot\left(\frac{\vec{q}}{m_{N}} \times \vec{v}^{\perp}\right) ; \quad O_{6}=\left(\vec{S}_{\chi} \cdot \frac{\vec{q}}{m_{N}}\right)\left(\vec{S}_{N} \cdot \frac{\vec{q}}{m_{N}}\right) ; \\
O_{7} & =\vec{S}_{N} \cdot \vec{v}^{\perp} ; \quad O_{8}=\vec{S}_{\chi} \cdot \vec{v}^{\perp} ; \quad O_{9}=i \vec{S}_{\chi} \cdot\left(\vec{S}_{N} \times \frac{\vec{q}}{m_{N}}\right) ; \\
O_{10} & =i \vec{S}_{N} \cdot \frac{\vec{q}}{m_{N}} ; \quad O_{11}=i \vec{S}_{\chi} \cdot \frac{\vec{q}}{m_{N}} ; \quad O_{12}=\vec{S}_{\chi} \cdot\left(\vec{S}_{N} \times \vec{v}^{\perp}\right) ; \\
O_{13} & =i\left(\vec{S}_{\chi} \cdot \vec{v}^{\perp}\right)\left(\vec{S}_{N} \cdot \frac{\vec{q}}{m_{N}}\right) ; \quad O_{14}=i\left(\vec{S}_{\chi} \cdot \frac{\vec{q}}{m_{N}}\right)\left(\vec{S}_{N} \cdot \vec{v}^{\perp}\right) ; \\
O_{15} & =-\left(\vec{S}_{\chi} \cdot \frac{\vec{q}}{m_{N}}\right)\left(\left(\vec{S}_{N} \times \vec{v}^{\perp}\right) \cdot \frac{\vec{q}}{m_{N}}\right) .
\end{aligned}
$$

In the above equation $1_{\chi N}$ is the identity operator, $\vec{q}$ is the transferred momentum, $\vec{S}_{\chi}$ and $\vec{S}_{N}$ are the WIMP and nucleon spins, respectively, while $\vec{v}^{\perp}=\vec{v}+\frac{\vec{q}}{2 \mu_{\chi N}}$ (with $\mu_{\chi N}$ the WIMPnucleon reduced mass) is the relative transverse velocity operator satisfying $\vec{v}^{\perp} \cdot \vec{q}=0$. Following Refs.[25, 26] in the following we will not include the operator $O_{2}$ in our analysis. For a nuclear target $T$ the quantity $\left(v_{T}^{\perp}\right)^{2} \equiv\left|\vec{v}_{T}^{\perp}\right|^{2}$ can also be written as:

$$
\left(v_{T}^{\perp}\right)^{2}=v_{T}^{2}-v_{\min }^{2} .
$$

where:

$$
v_{\min }^{2}=\frac{q^{2}}{4 \mu_{T}^{2}}=\frac{m_{T} E_{R}}{2 \mu_{T}^{2}}
$$

represents the minimal incoming WIMP speed required to impart the nuclear recoil energy $E_{R}$, while $v_{T} \equiv\left|\vec{v}_{T}\right|$ is the WIMP speed in the reference frame of the nuclear center of mass, $m_{T}$ the nuclear mass and $\mu_{T}$ the WIMP-nucleus reduced mass. Moreover $t^{0}=1, t^{1}=\tau_{3}$ denote the $2 \times 2$ identity and third Pauli matrix in isospin space, respectively, and the isoscalar and isovector (dimension -2) coupling constants $c_{j}^{0}$ and $c_{j}^{1}$, are related to those to protons and neutrons $c_{j}^{p}$ and $c_{j}^{n}$ by $c_{j}^{p}=\left(c_{j}^{0}+c_{j}^{1}\right)$ and $c_{j}^{n}=\left(c_{j}^{0}-c_{j}^{1}\right)$.

In the following we will only consider a contact effective interaction between the WIMP and the nucleus, i.e., we will assume the coefficients $c_{j}^{\tau}$ as independent on the transferred momentum $q$. However when the latter is comparable to the pion mass a pole is known to arise in the case of pseudoscalar and axial interactions [35]. This may affect our estimation of the sensitivity by less than an order of magnitude for operators $O_{6}$ and $O_{10}$ when the WIMP and the target mass are heavy (specifically, for xenon in XENON1T and PANDAX-II and for iodine in PICO-60). Since such effect depends on the particular relativistic model the NR theory descends from [36] and its impact is anyway limited, we have neglected it in our analysis.

The expected rate in a given visible energy bin $E_{1}^{\prime} \leq E^{\prime} \leq E_{2}^{\prime}$ of a direct detection experiment is given by:

$$
\begin{aligned}
R_{\left[E_{1}^{\prime}, E_{2}^{\prime}\right]} & =M \mathrm{~T} \int_{E_{1}^{\prime}}^{E_{2}^{\prime}} \frac{d R}{d E^{\prime}} d E^{\prime} \\
\frac{d R}{d E^{\prime}} & =\sum_{T} \int_{0}^{\infty} \frac{d R_{\chi T}}{d E_{e e}} \mathcal{G}_{T}\left(E^{\prime}, E_{e e}\right) \epsilon\left(E^{\prime}\right) d E_{e e} \\
E_{e e} & =q\left(E_{R}\right) E_{R},
\end{aligned}
$$


with $\epsilon\left(E^{\prime}\right) \leq 1$ the experimental efficiency/acceptance. In the equations above $E_{R}$ is the recoil energy deposited in the scattering process (indicated in keVnr), while $E_{e e}$ (indicated in keVee) is the fraction of $E_{R}$ that goes into the experimentally detected process (ionization, scintillation, heat) and $q\left(E_{R}\right)$ is the quenching factor, $\mathcal{G}_{\mathcal{T}}\left(E^{\prime}, E_{e e}=q\left(E_{R}\right) E_{R}\right)$ is the probability that the visible energy $E^{\prime}$ is detected when a WIMP has scattered off an isotope $T$ in the detector target with recoil energy $E_{R}, M$ is the fiducial mass of the detector and $\mathrm{T}$ the live-time of the data taking. For a given recoil energy imparted to the target the differential rate for the WIMP-nucleus scattering process is given by:

$$
\frac{d R_{\chi T}}{d E_{R}}(t)=\sum_{T} N_{T} \frac{\rho_{\mathrm{WIMP}}}{m_{\chi}} \int_{v_{\min }} d^{3} v_{T} f\left(\vec{v}_{T}, t\right) v_{T} \frac{d \sigma_{T}}{d E_{R}},
$$

where $\rho_{\text {WIMP }}$ is the local WIMP mass density in the neighborhood of the Sun, $N_{T}$ the number of the nuclear targets of species $T$ in the detector (the sum over $T$ applies in the case of more than one nuclear isotope), while

$$
\frac{d \sigma_{T}}{d E_{R}}=\frac{2 m_{T}}{4 \pi v_{T}^{2}}\left[\frac{1}{2 j_{\chi}+1} \frac{1}{2 j_{T}+1}\left|\mathcal{M}_{T}\right|^{2}\right],
$$

and, assuming that the nuclear interaction is the sum of the interactions of the WIMPs with the individual nucleons in the nucleus:

$$
\frac{1}{2 j_{\chi}+1} \frac{1}{2 j_{T}+1}\left|\mathcal{M}_{T}\right|^{2}=\frac{4 \pi}{2 j_{T}+1} \sum_{\tau=0,1} \sum_{\tau^{\prime}=0,1} \sum_{k} R_{k}^{\tau \tau^{\prime}}\left[c_{j}^{\tau},\left(v_{T}^{\perp}\right)^{2}, \frac{q^{2}}{m_{N}^{2}}\right] W_{T k}^{\tau \tau^{\prime}}(y) .
$$

In the above expression $j_{\chi}$ and $j_{T}$ are the WIMP and the target nucleus spins, respectively, $q=|\vec{q}|$ while the $R_{k}^{\tau \tau^{\prime}}$ 's are WIMP response functions (that we report for completeness in Eq. A.1p) which depend on the couplings $c_{j}^{\tau}$ as well as the transferred momentum $\vec{q}$ and $\left(v_{T}^{\perp}\right)^{2}$. In equation 12 the $W_{T k}^{\tau \tau^{\prime}}(y)$ 's are nuclear response functions and the index $k$ represents different effective nuclear operators, which, crucially, under the assumption that the nuclear ground state is an approximate eigenstate of $P$ and $C P$, can be at most eight: following the notation in [25, 26], $k=M, \Phi^{\prime \prime}, \Phi^{\prime \prime} M, \tilde{\Phi}^{\prime}, \Sigma^{\prime \prime}, \Sigma^{\prime}, \Delta, \Delta \Sigma^{\prime}$. The $W_{T k}^{\tau \tau^{\prime}}(y)$ 's are function of $y \equiv(q b / 2)^{2}$, where $b$ is the size of the nucleus. For the target nuclei $T$ used in most direct detection experiments the functions $W_{T k}^{\tau \tau^{\prime}}(y)$, calculated using nuclear shell models, have been provided in Refs. [26, 37] under the assumption that the dark matter particle couples to the nucleus through local onebody interactions with the nucleons. In our analysis we do not include two-body effects [38, 39] which are only available for a few isotopes and can be important when the one-body contribution is suppressed.

In the present paper, we will systematically consider the possibility that one of the couplings $c_{j}$ dominates in the effective Hamiltonian of Eq. (3). In this case it is possible to factorize a term $\left|c_{j}^{p}\right|^{2}$ from the squared amplitude of Eq. 12 and express it in terms of the effective WIMP-proton cross section 3

$$
\sigma_{p}=\left(c_{j}^{p}\right)^{2} \frac{\mu_{\chi N}^{2}}{\pi}
$$

\footnotetext{
${ }^{3}$ With the definition of Eq. 13 the WIMP-proton SI cross section is equal to $\sigma_{p}$, and the SD WIMP-proton cross section to $3 / 16 \sigma_{p}$.
} 
(with $\mu_{\chi \mathcal{N}}$ the WIMP-nucleon reduced mass) and the ratio $r \equiv c_{j}^{n} / c_{j}^{p}$. It is worth pointing out here that among the generalized nuclear response functions arising from the effective Hamiltonian of Eq. (3) only the ones corresponding to $M$ (SI interaction), $\Sigma^{\prime \prime}$ and $\Sigma^{\prime}$ (both related to the standard spin-dependent interaction) do not vanish for $q \rightarrow 0$, and so allow to interpret $\sigma_{p}$ in terms of a long-distance, point-like cross section. In the case of the other interactions $\Phi^{\prime \prime}, \Phi^{\prime \prime} M, \tilde{\Phi}^{\prime}, \Delta$ and $\Delta \Sigma^{\prime}$ the quantity $\sigma_{p}$ is just a convenient alternative to directly parameterizing the interaction in terms of the $c_{j}^{p}$ coupling.

Finally, $f\left(\vec{v}_{T}\right)$ is the WIMP velocity distribution, for which we assume a standard isotropic Maxwellian at rest in the Galactic rest frame truncated at the escape velocity $u_{e s c}$, and boosted to the Lab frame by the velocity of the Earth. So for the former we assume:

$$
\begin{aligned}
f\left(\vec{v}_{T}, t\right) & =N\left(\frac{3}{2 \pi v_{r m s}^{2}}\right)^{3 / 2} e^{-\frac{3\left|\vec{v}_{T}+\vec{v}_{E}\right|^{2}}{2 v_{r m s}^{2}}} \Theta\left(u_{e s c}-\left|\vec{v}_{T}+\vec{v}_{E}(t)\right|\right), \\
N & =\left[\operatorname{erf}(z)-\frac{2}{\sqrt{\pi}} z e^{-z^{2}}\right]^{-1},
\end{aligned}
$$

with $z=3 u_{e s c}^{2} /\left(2 v_{r m s}^{2}\right)$. In the isothermal sphere model hydrothermal equilibrium between the WIMP gas pressure and gravity is assumed, leading to $v_{r m s}=\sqrt{3 / 2} v_{0}$ with $v_{0}$ the galactic rotational velocity.

With the exception of DAMA, all the experiments included in our analysis are sensitive to the time average of the expected rate for which $\left\langle v_{E}\right\rangle=v_{\odot}$ and $v_{\odot}=v_{0}+12$ (accounting for a peculiar component of the solar system with respect to the galactic rotation). In the case of DAMA, the yearly modulation effect is due to the time dependence of the Earth's speed with respect to the Galactic frame, given by:

$$
\left|\vec{v}_{E}(t)\right|=v_{\odot}+v_{o r b} \cos \gamma \cos \left[\frac{2 \pi}{T_{0}}\left(t-t_{0}\right)\right],
$$

where $\cos \gamma \simeq 0.49$ accounts for the inclination of the ecliptic plane with respect to the Galactic plane, $T_{0}=1$ year and $v_{\text {orb }}=2 \pi r_{\oplus} /\left(T_{0}\right) \simeq 29 \mathrm{~km} / \mathrm{sec}\left(r_{\oplus}=1\right.$ AU neglecting the small eccentricity of the Earth's orbit around the Sun).

In our analysis for the two parameters $v_{0}$ and $u_{\text {esc }}$ we take $v_{0}=220 \mathrm{~km} / \mathrm{sec}$ [40] and $u_{\text {esc }}=550$ $\mathrm{km} / \mathrm{sec}$ [41]. Our choice of parameters corresponds to a WIMP escape velocity in the lab rest frame $v_{e s c}^{l a b} \simeq 782 \mathrm{~km} / \mathrm{s}$. To make contact with other analyses, for the dark matter density in the neighborhood of the Sun we use $\rho_{\text {WIMP }}=0.3$, which is a standard value commonly adopted by experimental collaborations, although observations point to the slightly higher value $\rho_{\text {шIмP }}=0.43$ [42, 43]. Notice that direct detection experiments are only sensitive to the product $\rho_{\text {WIMP }} \sigma_{p}$, so the results of the next Section can be easily rescaled with $\rho_{\text {WIMP }}$.

\section{Analysis}

The current $90 \%$ C.L. exclusion plots to the effective WIMP-proton cross section $\sigma_{p}$ of Eq. (13) for the SI interaction of Eq.(1) (corresponding to the $O_{1}$ operator in Eq.(3)) are shown for the isoscalar case $c_{1}^{p}=c_{1}^{n}$ and for the full set of the DM search experiments that we include in our analysis in Fig 1 . The plot includes the latest available data from a total of 14 existing 

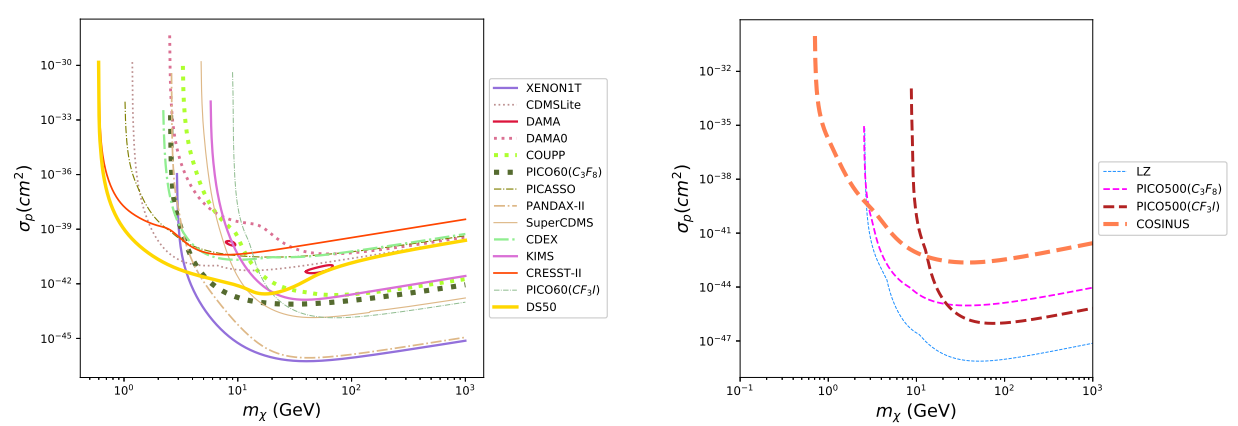

Figure 1: Current (left) and future (right) 90\% C.L. exclusion plots to the effective WIMP-proton cross section $\sigma_{p}$ of Eq. 13 for the SI interaction of Eq. (1) corresponding to the $O_{1}$ operator in Eq. 3 for the isoscalar case $c_{1}^{p}=c_{1}^{n}$. The figure shows the constraints from the full set of experiments that we include in our analysis, which consists in the latest available data from 14 existing DM searches, and the estimated future sensitivity of 4 projected ones (LZ, PICO-500 $\left(\mathrm{C}_{3} \mathrm{~F}_{8}\right)$, PICO-500 $\left(\mathrm{CF}_{3} \mathrm{I}\right)$ and COSINUS). The closed solid (red) contour represents the 5-sigma DAMA modulation amplitude region, while we indicate with DAMA0 the upper bound from the DAMA average count-rate. Notice that after the release of the DAMA/LIBRA-phase2 result [5] a spin-independent isoscalar $\left(c^{n} / c^{p}=1\right)$ interaction does not provide anymore a good fit to the modulation effect, while it still does for different values of $c^{n} / c^{p}$ and for other effective couplings [44].

experiments, and the estimated future sensitivity of 4 projected ones. The details of our procedure to obtain the exclusion plots are provided in Appendix B

The relative sensitivity of different detectors is determined by two elements: the thresholds $v_{\min }^{\text {th }}$ of different experiments expressed in terms of the WIMP incoming velocity, and the scaling law of the WIMP-nucleus cross section off different targets.

The former element explains the steep rise of all the exclusion plot curves at low WIMP masses, which corresponds to the case when $v_{\min }^{\text {th }}$ approaches the value of the escape velocity in the lab rest frame, and is sensitive to experimental features close to the energy threshold that are typically affected by uncertainties, such as efficiencies, acceptances and charge/light yields. With the assumptions listed in Appendix B, among the experiments included in our analysis the ones with the lowest velocity thresholds turn out to be DS50, CRESST-II, CDMSlite and CDEX. In particular, for $m_{\chi}=1 \mathrm{GeV}$ we have $v_{\min , D S 50}^{\text {th }} \simeq 450 \mathrm{~km} / \mathrm{s}, v_{\min , \text { CRESST-II }}^{\text {th }} \simeq 480 \mathrm{~km} / \mathrm{s}$ (for scatterings off oxygen), $v_{\text {min,CDMSlite }}^{\text {th }} \simeq 910 \mathrm{~km} / \mathrm{s}, v_{\text {min, CDEX }}^{\text {th }} \simeq 1600 \mathrm{~km} / \mathrm{s}$. Assuming $v_{e s c}^{\text {lab }} \simeq$ $782 \mathrm{~km} / \mathrm{s}$ (see the previous Section) this implies that in our analysis only DS50 and CRESST-II (for effective interactions for which argon and oxygen have a non-vanishing nuclear response function) are sensitive to $m_{\chi} \lesssim 1 \mathrm{GeV}$. On the other hand CDMSlite and CDEX are sensitive to slightly higher masses (for instance, for $m_{\chi}=2 \mathrm{GeV} v_{\text {min,CDMSlite }}^{\text {th }} \simeq 460 \mathrm{~km} / \mathrm{s}, v_{\text {min,CDEX }}^{\text {th }} \simeq 850$ $\mathrm{km} / \mathrm{s}$, while for $m_{\chi}=3 \mathrm{GeV} v_{\min , C D E X}^{\text {th }} \simeq 580 \mathrm{~km} / \mathrm{s}$ ). The velocity threshold is a purely kinematical feature that does not depend on the type of interaction and that favors experiments with the lowest $v_{\text {min }}^{\text {th }}$ at fixed $m_{\chi}$.

With the exception of very low masses, where the effect of $v_{\min }^{\text {th }}$ is dominant, the relative sensitivity of different detectors is determined by the scaling law of the WIMP-nucleus cross section with different targets, which is the focus of our analysis. In particular the SI interaction (corresponding to the $M$ effective nuclear operator) favors heavy nuclei, so that the most stringent 


\begin{tabular}{|c|c|c|c|c|c|}
\hline coupling & $R_{0 k}^{\tau \tau^{\prime}}$ & $R_{1 k}^{\tau \tau^{\prime}}$ & coupling & $R_{0 k}^{\tau \tau^{\prime}}$ & $R_{1 k}^{\tau \tau^{\prime}}$ \\
\hline 1 & $M\left(q^{0}\right)$ & - & 3 & $\Phi^{\prime \prime}\left(q^{4}\right)$ & $\Sigma^{\prime}\left(q^{2}\right)$ \\
4 & $\Sigma^{\prime \prime}\left(q^{0}\right), \Sigma^{\prime}\left(q^{0}\right)$ & - & 5 & $\Delta\left(q^{4}\right)$ & $M\left(q^{2}\right)$ \\
6 & $\Sigma^{\prime \prime}\left(q^{4}\right)$ & - & 7 & - & $\Sigma^{\prime}\left(q^{0}\right)$ \\
8 & $\Delta\left(q^{2}\right)$ & $M\left(q^{0}\right)$ & 9 & $\Sigma^{\prime}\left(q^{2}\right)$ & - \\
10 & $\Sigma^{\prime \prime}\left(q^{2}\right)$ & - & 11 & $M\left(q^{2}\right)$ & - \\
12 & $\Phi^{\prime \prime}\left(q^{2}\right), \tilde{\Phi}^{\prime}\left(q^{2}\right)$ & $\Sigma^{\prime \prime}\left(q^{0}\right), \Sigma^{\prime}\left(q^{0}\right)$ & 13 & $\tilde{\Phi}^{\prime}\left(q^{4}\right)$ & $\Sigma^{\prime \prime}\left(q^{2}\right)$ \\
14 & - & $\Sigma^{\prime}\left(q^{2}\right)$ & 15 & $\Phi^{\prime \prime}\left(q^{6}\right)$ & $\Sigma^{\prime}\left(q^{4}\right)$ \\
\hline
\end{tabular}

Table 1: Nuclear response functions corresponding to each coupling, for the velocity-independent and the velocitydependent components parts of the WIMP response function, decomposed as in Eq. 17. In parenthesis the power of $q$ in the WIMP response function.

bounds in Fig. 11 correspond to xenon experiments (XENON1T, PANDAX-II). However the interaction terms in the Hamiltonian of Eq. (3) lead to expected rates that depend on the full set of possible nuclear operators $\left(M, \Phi^{\prime \prime}, \tilde{\Phi}^{\prime}, \Sigma^{\prime \prime}, \Sigma^{\prime}, \Delta\right)$ leading to different scaling laws of the WIMP-nucleus cross section on different targets. The correspondence between models and nuclear response functions can be directly read off from the WIMP response functions $R_{k}^{\tau \tau^{\prime}}$ (see Eq A.1. In particular, using the decomposition:

$$
R_{k}^{\tau \tau^{\prime}}=R_{0 k}^{\tau \tau^{\prime}}+R_{1 k}^{\tau \tau^{\prime}}\left(v_{T}^{\perp}\right)^{2}=R_{0 k}^{\tau \tau^{\prime}}+R_{1 k}^{\tau \tau^{\prime}}\left(v_{T}^{2}-v_{\text {min }}^{2}\right),
$$

such correspondence is summarized in Table 1] In Fig. 2 we provide for completeness the nuclear response functions at vanishing momentum transfer off protons $16 \pi /\left(2 j_{T}+1\right) \times W_{T k}^{p}(y=0)$ (lefthand plot) and off neutrons $16 \pi /\left(2 j_{T}+1\right) \times W_{T k}^{n}(y=0)$ (right-hand plot), with $W_{T k}^{p, n} \equiv 1 / 4 \times\left(W_{T k}^{00} \pm\right.$ $\left.W_{T k}^{01} \pm W_{T k}^{10}+W_{T k}^{11}\right)$ for $k=M, \Phi^{\prime \prime}, \tilde{\Phi}^{\prime}, \Sigma^{\prime \prime}, \Sigma^{\prime}, \Delta$ and all the targets $T$ used in the present analysis, as calculated in [26, 37]. The normalization factor is chosen so that $16 \pi /\left(2 j_{T}+1\right) \times W_{T M}^{p}(y=0)=Z_{T}^{2}$ and $16 \pi /\left(2 j_{T}+1\right) \times W_{T M}^{n}(y=0)=\left(A_{T}-Z_{T}\right)^{2}$ with $A_{T}, Z_{T}$ the mass and atomic numbers for target $T$. In the same figure values below the horizontal line at $1 \times 10^{-4}$ represent nuclear response functions that are missing in the literature. They enter in the calculation of expected rates in KIMS (caesium, using CsI) and CRESST-II (tungsten, using $\mathrm{CaWO}_{4}$ ). In both cases we have calculated the expected rate on the targets with known nuclear response functions and set to zero the missing ones, so that the corresponding constraints must be considered as conservative estimates. For the targets for which Refs. [26, 37] do not provide the nuclear response functions we evaluate the standard SI and SD interactions following the procedure of Appendix C

The sensitivity of present experiments to each of the couplings of the effective Hamiltonian of Eq. (3) is discussed in Figs. 3 16, which show the contour plots of the most stringent $90 \%$ C.L. bound on the effective WIMP-nucleon cross section $\sigma_{\mathcal{N}}$, defined as:

$$
\sigma_{\mathcal{N}}=\max \left(\sigma_{p}, \sigma_{n}\right)
$$

as a function of the WIMP mass $m_{\chi}$ and of the ratio $c^{n} / c^{p}$ between the WIMP-neutron and the WIMP-proton couplings. The numerical values in the figures indicate the most stringent bound on $\sigma_{\mathcal{N}}$ in $\mathrm{cm}^{2}$. In each plot the different shadings (colors) indicate the experiment providing the most constraining bound, as indicated in the corresponding legend. To make such plots of practical use, in Appendix D we introduce NRDD_constraints, a simple interpolating code written in Python that allows to extract the numerical values of $\sigma_{\mathcal{N}}$ from Figs. 3 16 In the calculation 

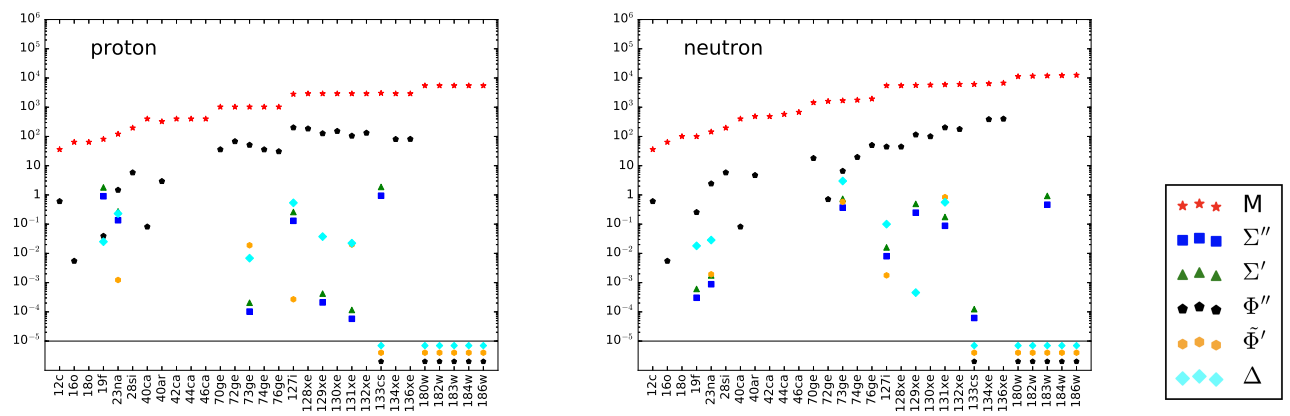

Figure 2: Nuclear response functions at vanishing momentum transfer off protons $16 \pi /\left(2 j_{T}+1\right) \times W_{T k}^{p}(y=0)($ lefthand plot) and off neutrons $16 \pi /\left(2 j_{T}+1\right) \times W_{T k}^{n}(y=0)$ (right-hand plot), for $k=M, \Phi^{\prime \prime}, \tilde{\Phi}^{\prime}, \Sigma^{\prime \prime}, \Sigma^{\prime}, \Delta$ and all the targets $T$ used in the present analysis. The normalization factor is chosen so that $16 \pi /\left(2 j_{T}+1\right) \times W_{T M}^{p}(y=0)=Z_{T}^{2}$ and $16 \pi /\left(2 j_{T}+1\right) \times W_{T M}^{n}(y=0)=\left(A_{T}-Z_{T}\right)^{2}$ with $A_{T}, Z_{T}$ the mass and atomic numbers for target $T$. Markers below the horizontal solid line represent nuclear response functions that are missing in the literature. In our analysis we have set them to zero.

of all the plots the lower part of the 2-sigma DAMA modulation amplitude region in the $m_{\chi}-\sigma_{p}$ plane is included as if it were an additional constraint, in order to locate possible regions of compatibility between the DAMA excess and other constraints in the parameter space. As can be seen from Figs. 3 16DAMA never appears as the most constraining bound, indicating that an explanation of its annual modulation excess in terms of a WIMP signal is in tension with the constraints of other experiments no matter which of the effective operators among those in Eq. (3) is assumed to dominate in the WIMP-nucleus interaction and for all $\left(m_{\chi}-\sigma_{\mathcal{N}}\right)$ combinations. This result is in agreement with the findings of Ref. [45, 44].

The different patterns of the regions appearing in Figs. $3-16$ can be understood with the help of Table 1 and Fig 2. In particular, the velocity-dependent contribution proportional to $\left(v_{T}^{\perp}\right)^{2}$ is negligible or absent in all cases with 5 exceptions: $c_{7}$ and $c_{14}$ (where the velocity-independent term is not present), $c_{5}$ and $c_{8}$ (where it is enhanced by the $M$ coherent response functions) and for the coupling $c_{13}$ (see below). As a consequence of this, the interaction terms $c_{1}, c_{5}$, $c_{8}$ and $c_{11}$ depend on the $M$ coherent response function with a consequent strong sensitivity of xenon detectors (XENON1T and PANDAX-II) except for $c^{n} / c^{p} \simeq-0.7$ corresponding to a suppression on xenon targets. By the same token, the interactions terms $c_{4}, c_{6}, c_{7}, c_{9}, c_{10}$ and $c_{14}$ depend on the response functions $\Sigma^{\prime \prime}$ and/or $\Sigma^{\prime}$, that are related to the spin-dependent coupling of Eq. 22): in particular, $\Sigma^{\prime \prime}$ corresponds to the coupling of the WIMP to the component of the nucleon spin along the direction of the transferred momentum $\vec{q}$ while $\Sigma^{\prime}$ to that perpendicular to it, with $W_{\Sigma^{\prime}}^{\tau \tau^{\prime}}\left(q^{2}\right) \simeq 2 W_{\Sigma^{\prime \prime}}^{\tau \tau^{\prime}}\left(q^{2}\right)$ when $q^{2} \rightarrow 0$. Since inside nuclei the nucleons spins tend to cancel each other the contribution from even-numbered nucleons to the response functions $\Sigma^{\prime \prime}$ and $\Sigma^{\prime}$ is strongly suppressed. As a consequence of this for such interactions neutron-odd targets (such as xenon and germanium) are mostly sensitive to the regime $\left|c^{n} / c^{p}\right| \gtrsim 1$ while proton-odd ones (such as fluorine and iodine) mainly constrain the opposite case $\left|c^{n} / c^{p}\right| \lesssim 1$. This reflects in the pattern of the shaded areas of Figs. 5, 7, 8, 10, 11 and 15, where for $m_{\chi} \gtrsim 1 \mathrm{GeV}$ the PICASSO and $\mathrm{PICO}\left(\mathrm{C}_{3} \mathrm{~F}_{8}\right)$ bounds (using proton-odd fluorine) are the most constraining limits for $\left|c^{n} / c^{p}\right| \lesssim 1$ (with the exception of $c_{6}$, where also $\mathrm{PICO}\left(\mathrm{CF}_{3} \mathrm{I}\right)$ becomes competitive in spite 
of the relatively large energy threshold $\left(E_{R}=13.6 \mathrm{keV}\right)$ due to the $q^{4}$ momentum dependence that enhances the Iodine nuclear response function), while XENON1T and PANDAX-II (using neutron-odd xenon) drive the constraints for $\left|c^{n} / c^{p}\right| \gtrsim 1$. On the other hand, at lower masses the constraint is driven by CDMSlite, which is the experiment with a non-vanishing spin target (germanium) which has the lowest energy threshold (in particular DS50 and CRESST-II do not put any constraint in this regime since argon and oxygen are spinless nuclei). One can also notice in Figs. 8 and 15 the loss of sensitivity of PICASSO at low WIMP mass $\left(m_{\chi} \lesssim 5 \mathrm{GeV}\right)$ for the velocity-dependent couplings $c_{7}$ and $c_{14}$ compared to the case of other spin-dependent couplings: this is due to the fact that the constraint from PICASSO is driven by its low energy threshold $\left(E_{t h} \simeq 1 \mathrm{keV}\right)$ and consequent low $v_{\min }$ : however, for a velocity-dependent cross section the contribution to the rate of the part of the velocity integral close to $v_{\min }$ is suppressed by the term $v^{2}-v_{\min }^{2}$, weakening the corresponding bound.

Another set of plots with a similar color pattern is given by Figs, 4, 13 and 16 , corresponding to the couplings $c_{3}, c_{12}$ and $c_{15}$. As can be seen from Table 1 in all these cases expected rates are driven by the $\Phi^{\prime \prime}$ nuclear response function, which is related to spin-orbit coupling $\vec{\sigma} \cdot \vec{l}[25]$. Such response function is non vanishing for all nuclei and favors heavier elements with large nuclear shell model orbitals not fully occupied and, as can be seen from Fig,2, its scaling with the nuclear target is similar to the SI interaction (albeit the corresponding nuclear response functions are about two orders of magnitude smaller), with XENON1T the most constraining experiment at large-enough WIMP masses and DS50 constraining the low-mass range due to its low velocity threshold. In all the three figures one can also observe a region for $2 \mathrm{GeV} \lesssim m_{\chi} \lesssim 4 \mathrm{GeV}$ and $\left|c^{n}\right|<\left|c^{p}\right|$ where the CDMSlite constraint becomes competitive with DS50. Indeed, in this range of masses the two constraints are quite close in all the range of $c^{n} / c^{p}$, with a slight weakening of the CDMSlite bound for $\left|c^{n}\right|>\left|c^{p}\right|$ due to the suppressed response off neutrons in the semi-magic isotope ${ }^{72} \mathrm{Ge}$ (the dot product $\vec{\sigma} \cdot \vec{l}$ vanishes for completely filled angular momentum orbitals [25]), as can be seen in the right-hand plot of Fig 2 .

The case of the coupling $c_{13}$ shown in Fig.14 is the only one that depends in a sizable way on the nuclear response function $\tilde{\Phi}^{\prime}$, which is related to a vector-longitudinal operator that transforms as a tensor under rotations [25, 26]. From the phenomenological point of view, such operator requires a nuclear spin $j>1 / 2$, so that, among the isotopes used in DM searches, it is non-vanishing only for the four isotopes ${ }^{23} \mathrm{Na},{ }^{73} \mathrm{Ge},{ }^{127} \mathrm{I}$ and ${ }^{131} \mathrm{Xe}$. Indeed, the most stringent constraints arise in this case from CDMSlite at low WIMP masses and from XENON1T at larger values. Nevertheless, in Fig. 14two fluorine detectors (PICASSO and PICO-60) yield the stronger constraints in the mass range $5 \mathrm{GeV} \lesssim m_{\chi} \lesssim 7 \mathrm{GeV}$ and for $\left|c^{n} / c^{p}\right|<1$. This is explained by the spin-dependent term with an explicit velocity dependence in the decomposition of Eq.[17p, that, in spite of the suppression due to the slow incoming WIMP speeds, can become as constraining as the velocity-independent coupling off xenon in XENON1T.

We conclude our discussion with a comment on the dependence of the direct detection signal on the recoil energy. Besides a different scaling of the cross section with the target, the nonstandard interactions listed in Table 1 involve cases where the cross section depends explicitly on the momentum transfer $q=\sqrt{2 m_{T} E_{R}}$, implying a harder energy spectrum of the expected signal compared to the usual exponentially decaying case observed for the standard SI and SD cases. This may lead to a weakening of the constraints compared to the standard case when, as for DS50 and KIMS (see Appendix B and Eq. (B.2p)), a background estimation growing with energy is subtracted from the data. Indeed, we observe this effect in our analysis, but it is significant only for WIMP masses large enough for the expected rate to be insensitive to the high-speed 
tail of the velocity distribution. On the other hand large count rates requiring background subtraction are typically present only in experiments that, such as DS50 and KIMS, focus on a low energy threshold to reach a competitive sensitivity at low WIMP masses at the expense of the efficiency of their background discrimination. For such low values of $m_{\chi}$ the signal spectrum has a steep decay with energy also in presence of a $q^{n}$ term in the cross section and the effectiveness of background subtraction is similar to the standard case. A dependence of the constraint on the expected signal spectral shape enters also in the optimal-interval method [46] that we have applied in the case of SuperCDMS.

The full set of bounds is summarized in Fig. 17,18 , where for each of the couplings of the Hamiltonian of Eq. (3) the most stringent constraint on $\sigma_{\mathcal{N} \text {,lim }}$ is plotted as a function of the WIMP mass $m_{\chi}$. In each plot the two curves indicated by "present min" and "present max" show the range of the most stringent limit at fixed $m_{\chi}$ on $\sigma_{\mathcal{N}, \text { lim }}$ from present experiments when the ratio $c^{n} / c^{p}$ is varied in the same interval of Figs 3 - 16 , while the curves indicated by "future min" and "future max" show the same range when the expected bound from some projected experiments (LUX-ZEPLIN (LZ), PICO-500 $\left(\mathrm{C}_{3} \mathrm{~F}_{8}\right)$, PICO-500 $\left(\mathrm{CF}_{3} \mathrm{I}\right)$ and COSINUS) are included (see Appendix B for details). The styles of each curve indicate the experiment providing the most stringent bound, as shown by the corresponding legend. Notice that at a given value of the WIMP mass an upper bound on the effective cross section $\sigma_{\mathcal{N}}$ corresponds to a lower bound on the effective coupling $c^{n, p}$ which, in case of a spin-1/2 particle, has dimension $\mathrm{GeV}^{-2}$. Writing $c^{n, p}=1 / M_{E F T}^{2}$ with $M_{E F T}$ a cut-off scale, the validity of the NR effective theory requires $M_{E F T} \gtrsim$ $1 \mathrm{GeV}$, which implies $\sigma_{\mathcal{N}} \lesssim 10^{-30} \mathrm{~cm}^{2}$. Some of the bounds on $\sigma_{\mathcal{N}}$ if Figs 17,18 and 19 are not compatible to such condition, especially for low WIMP masses where expected rates are suppressed by the velocity distribution. This can be simply interpreted as the fact that in such regimes present sensitivities do not pose any sensible bound on the corresponding coupling.

For each of the couplings of the effective Hamiltonian the most stringent bounds from present and future experiments on $\sigma_{\mathcal{N}, \text { lim }}$ are tabulated in Table 2, where the ratio $c^{n} / c^{p}$ is fixed in each case to the value that corresponds to the stronger constraint, and in Table 3 when $c^{n} / c^{p}$ corresponds to the weaker constraint. One can see that the expected reach on $\sigma_{\mathcal{N}, l i m}$ varies by many orders of magnitude with the effective coupling. For all the NR couplings we consider XENON1T yields the most constraining bound among existing experiments for WIMP masses below $1 \mathrm{TeV}$. Among future experiments LZ has the highest sensitivity at all WIMP masses for $c_{1}, c_{3}, c_{5}, c_{8}$, $c_{11}, c_{12}, c_{13}$ and $c_{15}$ while for other couplings either LZ or PICO-500 $\left(\mathrm{C}_{3} \mathrm{~F}_{8}\right)$ corresponds to the most constraining limit depending on $m_{\chi}$. On the other hand as shown in Figs. 33 16, 9 present experiments out of the total of 14 considered in our analysis provide the most stringent bound on some of the effective couplings for a given choice of $\left(m_{\chi}, c^{n} / c^{p}\right)$ : XENON1T, PANDAX-II, CDMSlite, PICASSO, PICO-60 $\left(\mathrm{CF}_{3} \mathrm{I}\right)$, PICO-60 $\left(\mathrm{C}_{3} \mathrm{~F}_{8}\right)$, CRESST-II, DAMA0 (average count rate) and DarkSide-50. This is evidence of the complementarity of different target nuclei and/or different combinations of count-rates and energy thresholds when the search of a DM particle is extended to a wide range of possible interactions. The variation of the best reach on $\sigma_{\mathcal{N} \text {, lim }}$ with $c^{n} / c^{p}$ is about 3 orders of magnitude for $c_{1}, c_{11}$ and $c_{13}$, about 2 orders of magnitude for $c_{8}$, between 1 and 2 orders of magnitude for $c_{3}, c_{5}, c_{12}$ and $c_{15}$, about one order of magnitude for $c_{6}, c_{10}$ and less than one order of magnitude for $c_{4}, c_{7}, c_{9}$ and $c_{14}$. For all couplings future experiments could improve the present best reach between two and three orders of magnitude. 

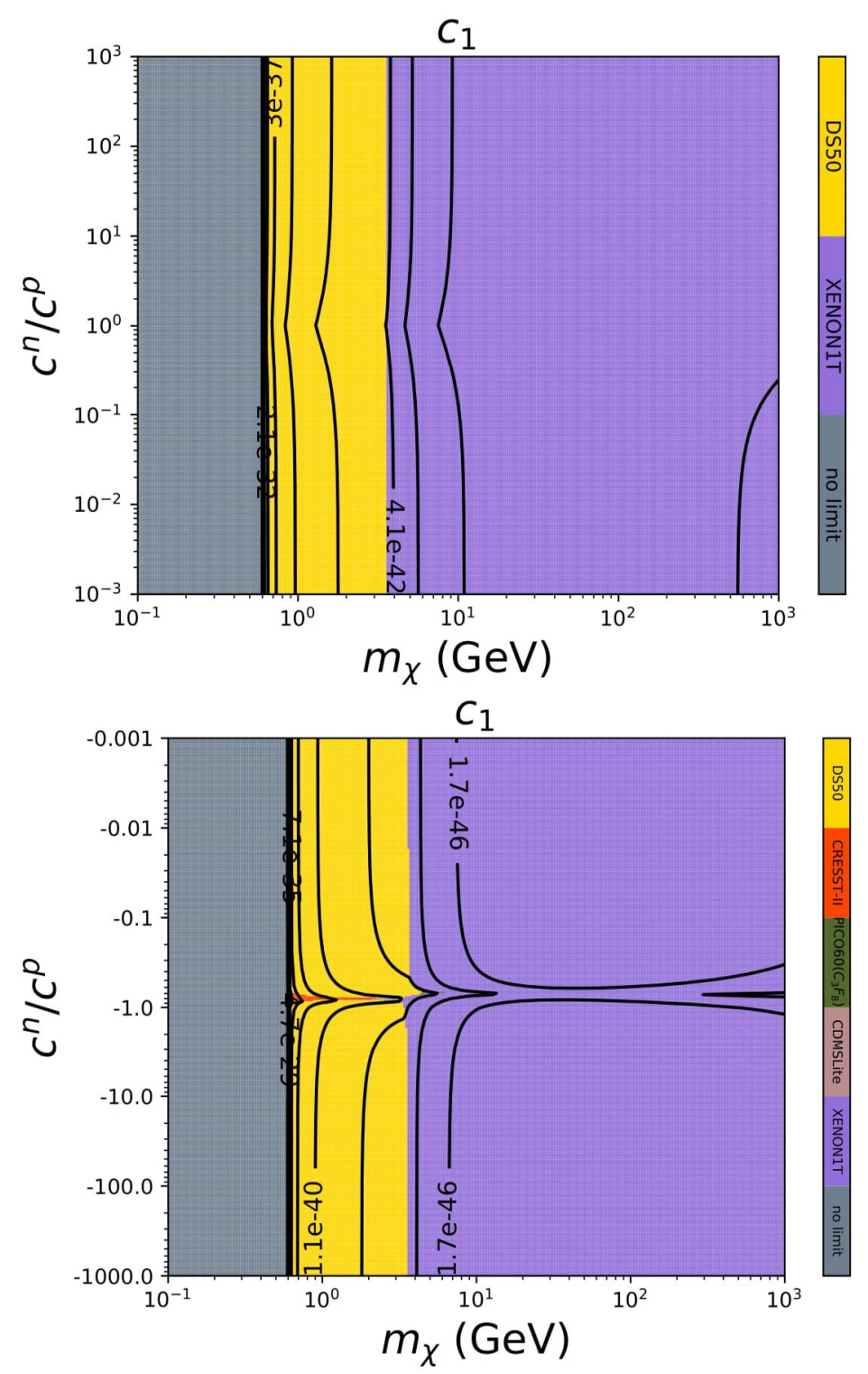

Figure 3: Contour plots of the most stringent bound on the effective cross section $\sigma_{\mathcal{N}, l i m}$ introduced in Eq. 18 as a function of the WIMP mass $m_{\chi}$ and of the ratio $c^{n} / c^{p}$ between the WIMP-neutron and the WIMP-proton couplings assuming that the operator $O_{1}$ dominates in the effective Hamiltonian of Eq. 3. Numerical values of the limit on $\sigma_{\mathcal{N}}$ are in $\mathrm{cm}^{2}$. Different shadings indicate the experiment providing the most constraining bound, as indicated in the legend. 

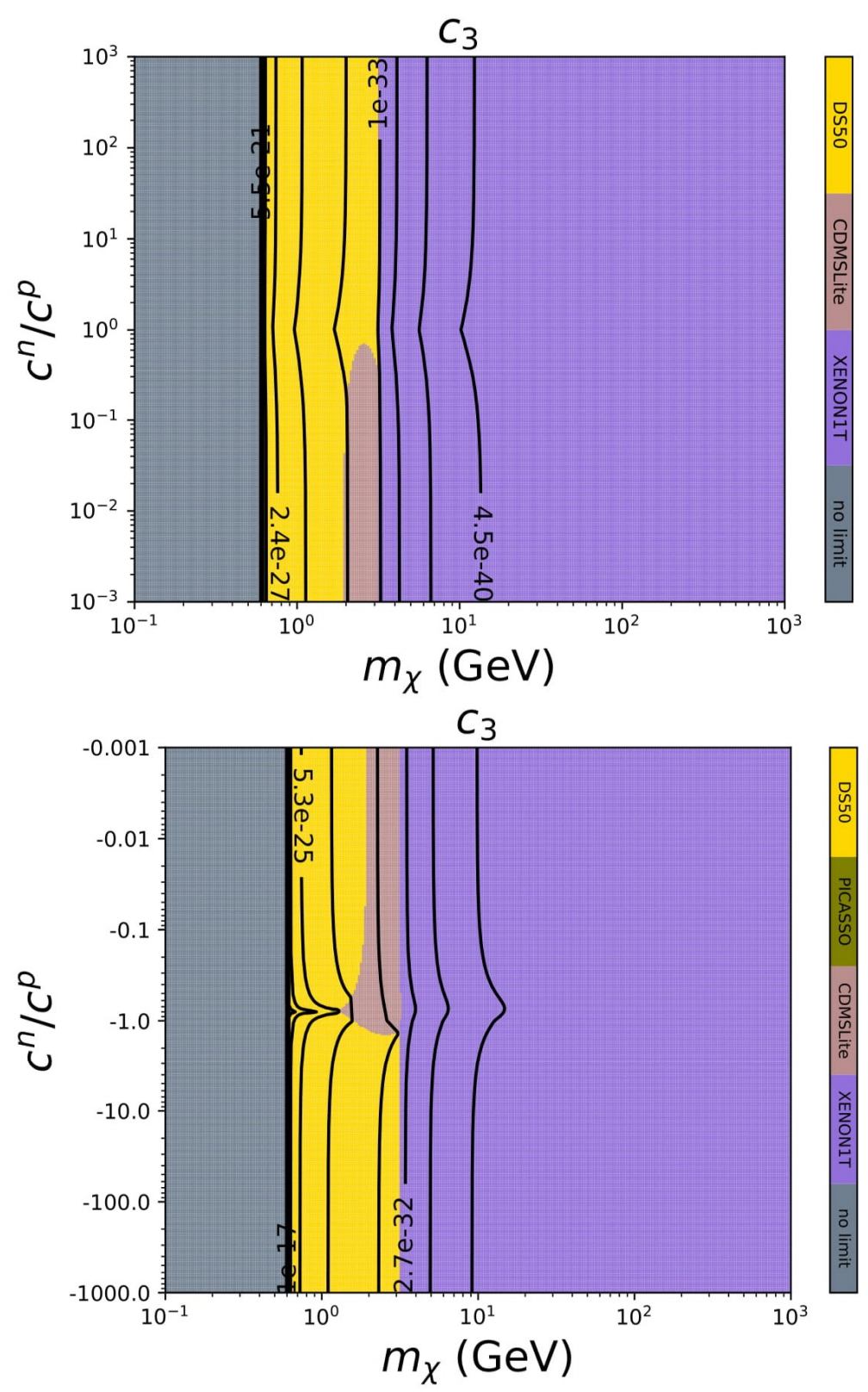

Figure 4: The same as in $\mathrm{Fig} 3$ for the operator $O_{3}$. 

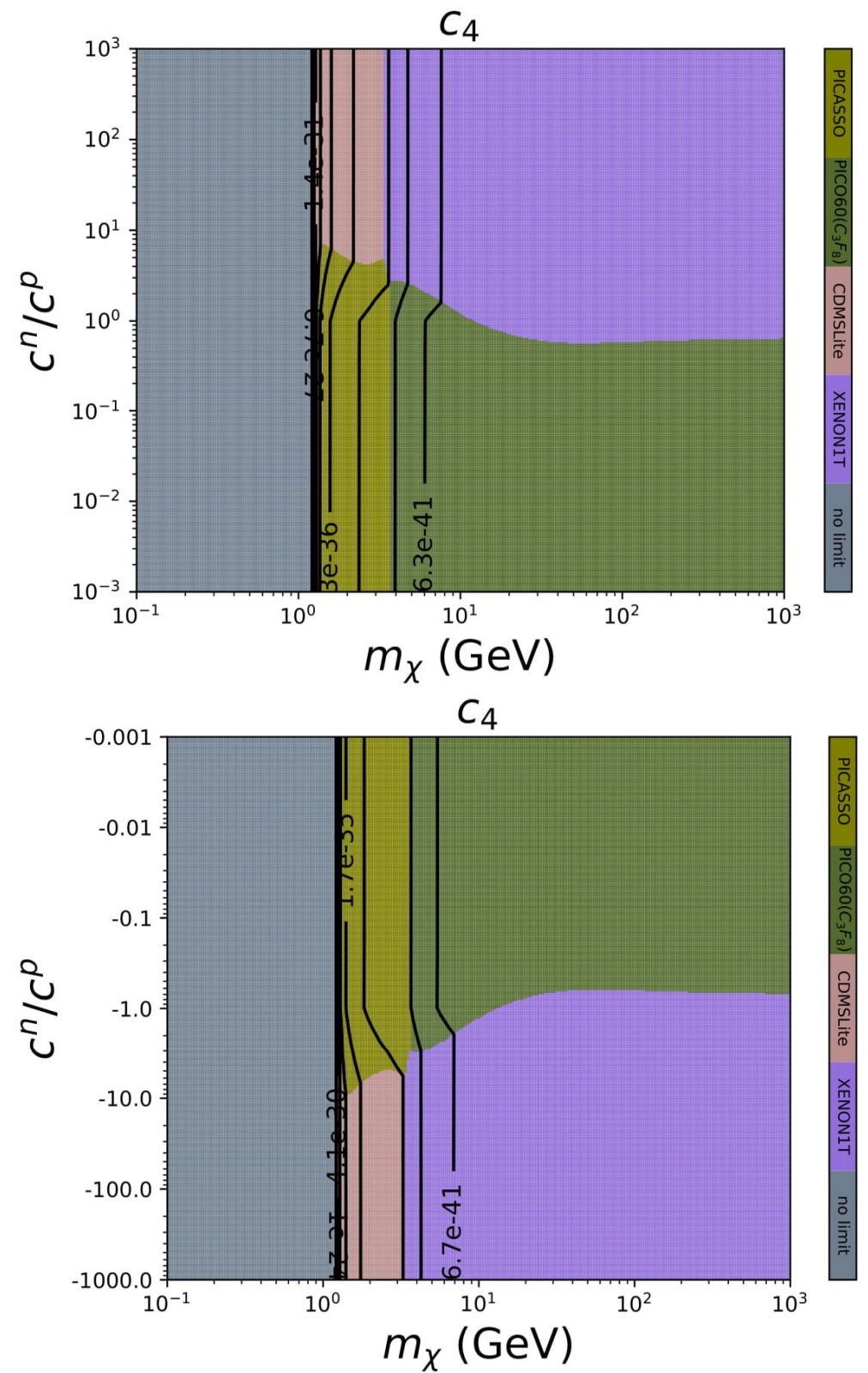

Figure 5: The same as in Fig 3 for the operator $O_{4}$. This operator corresponds to the standard spin-dependent interaction of Eq. 22. 

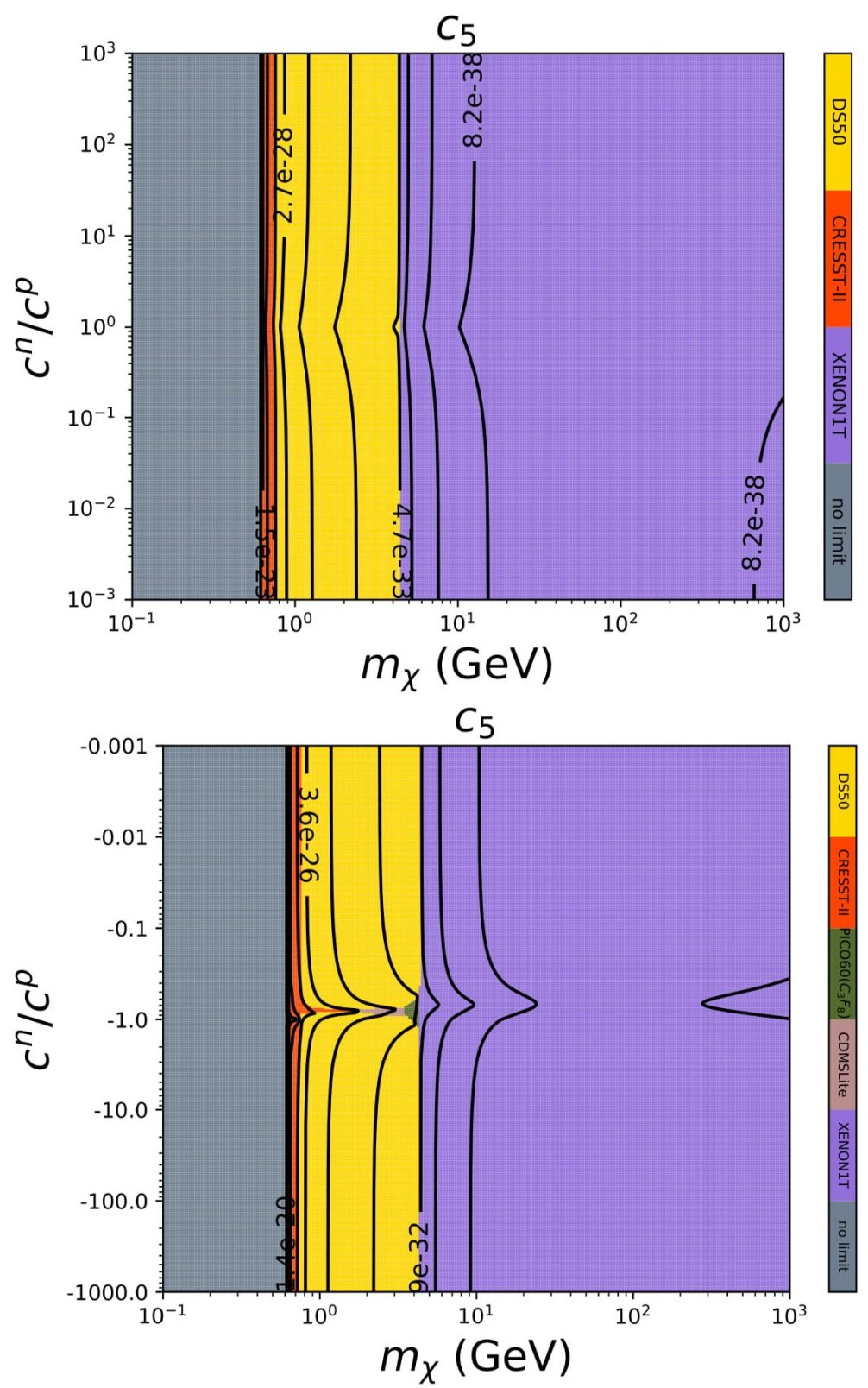

Figure 6: The same as in Fig 3 for the operator $O_{5}$. 

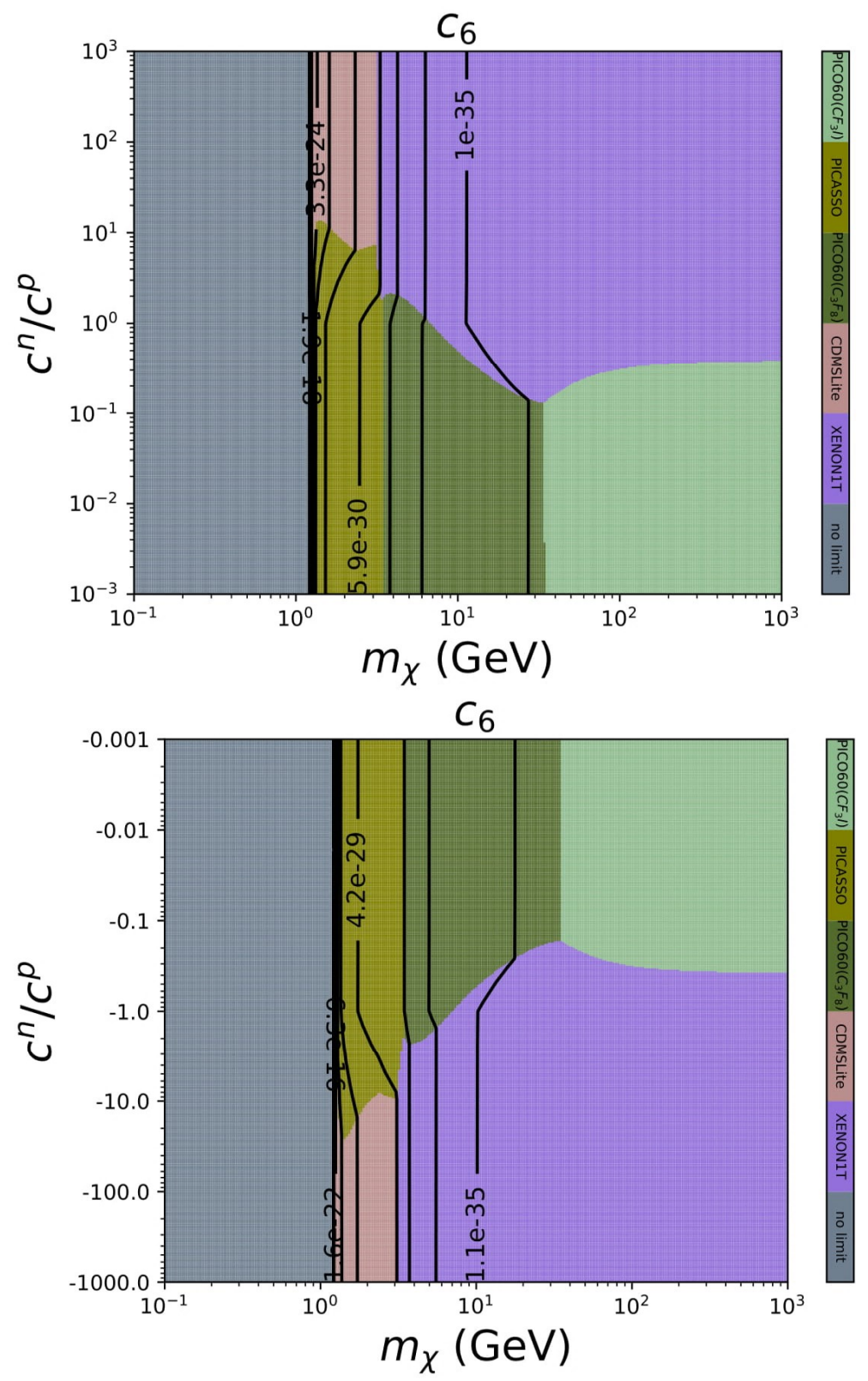

Figure 7: The same as in Fig 3 for the operator $O_{6}$. 

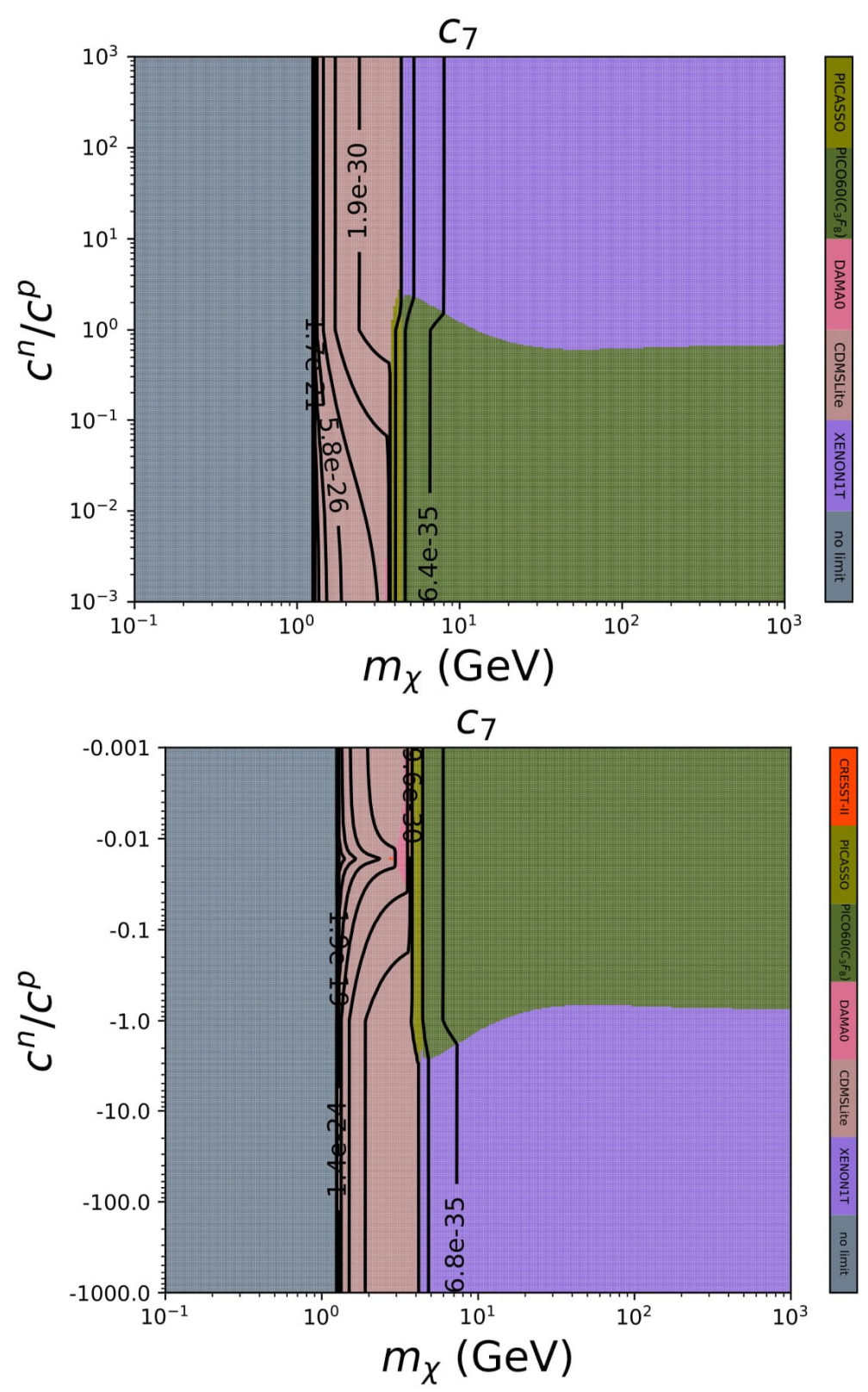

Figure 8: The same as in $\mathrm{Fig} 3$ for the operator $O_{7}$. 

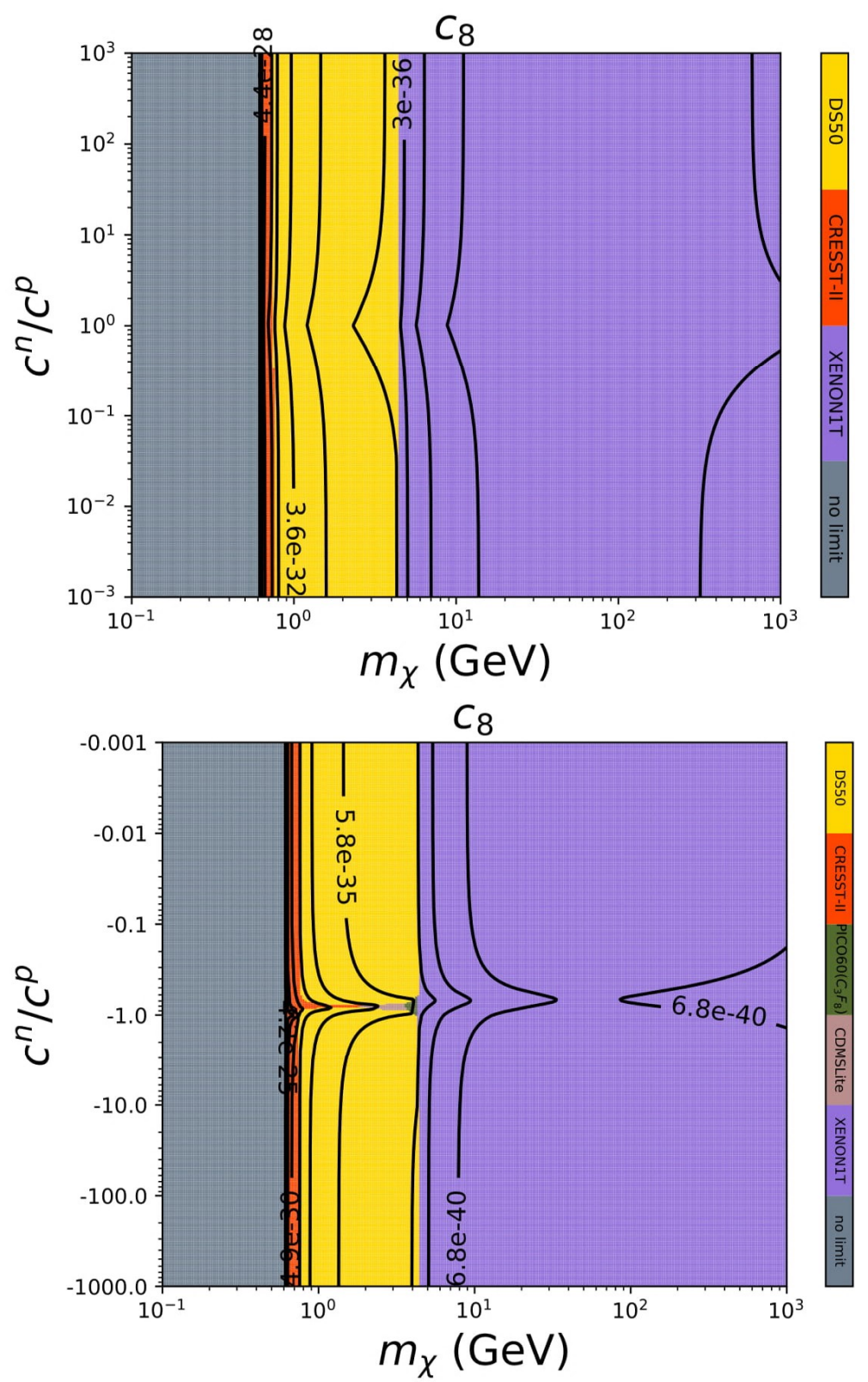

Figure 9: The same as in $\mathrm{Fig} 3$ for the operator $O_{8}$. 

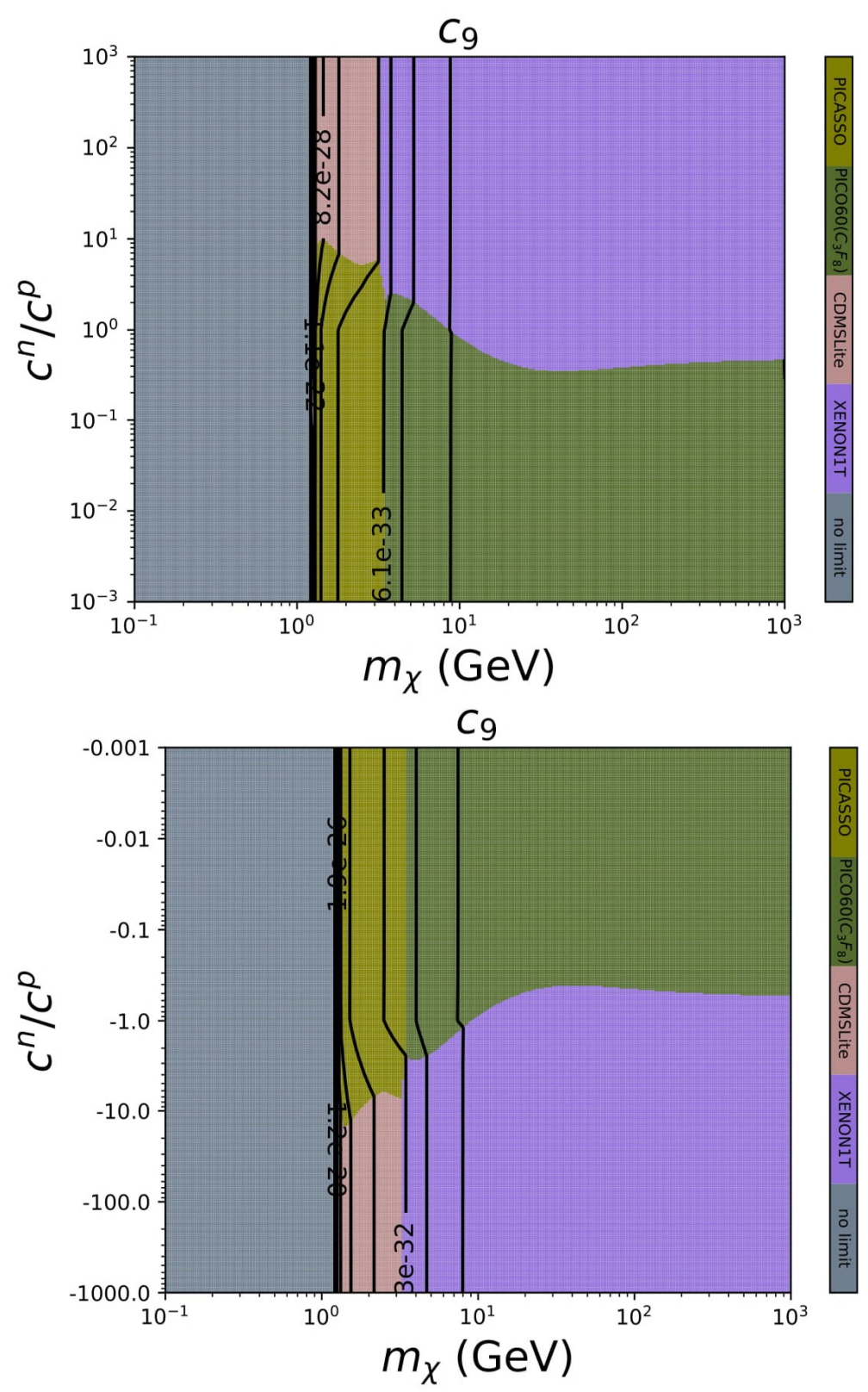

Figure 10: The same as in $\mathrm{Fig} 3$ for the operator $O_{9}$. 

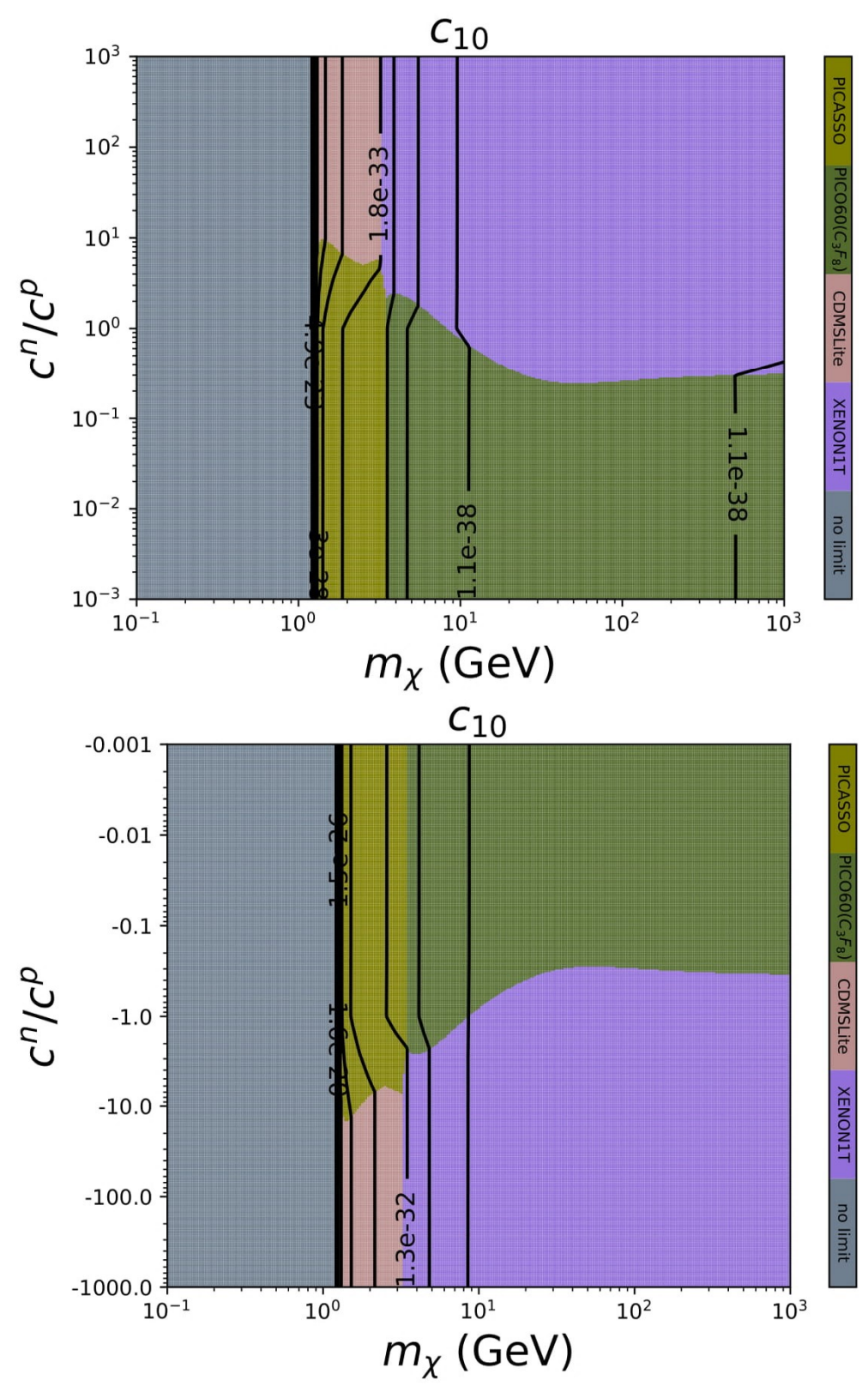

Figure 11: The same as in $\mathrm{Fig} 3$ for the operator $O_{10}$. 

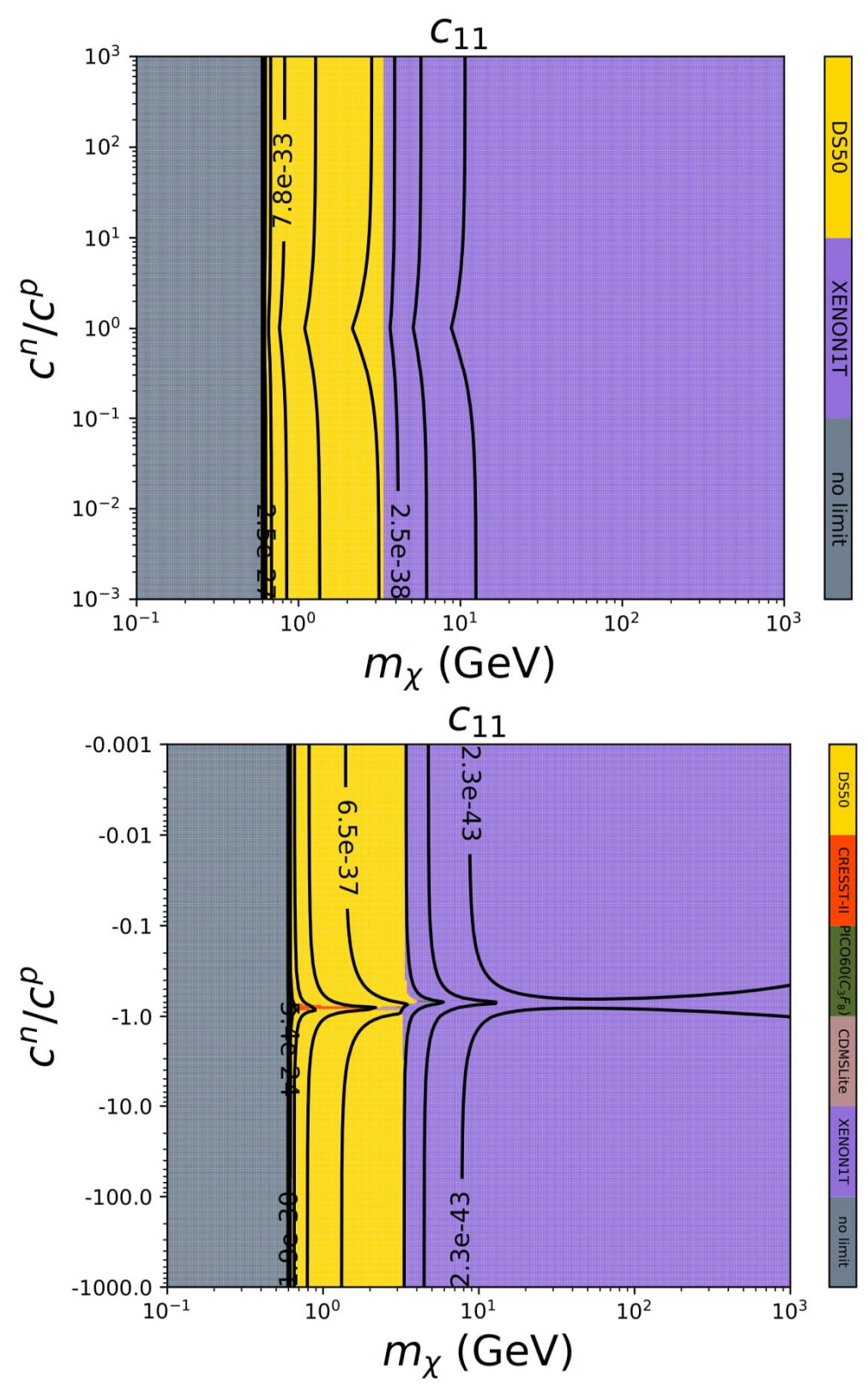

Figure 12: The same as in $\mathrm{Fig} 3$ for the operator $O_{11}$. 

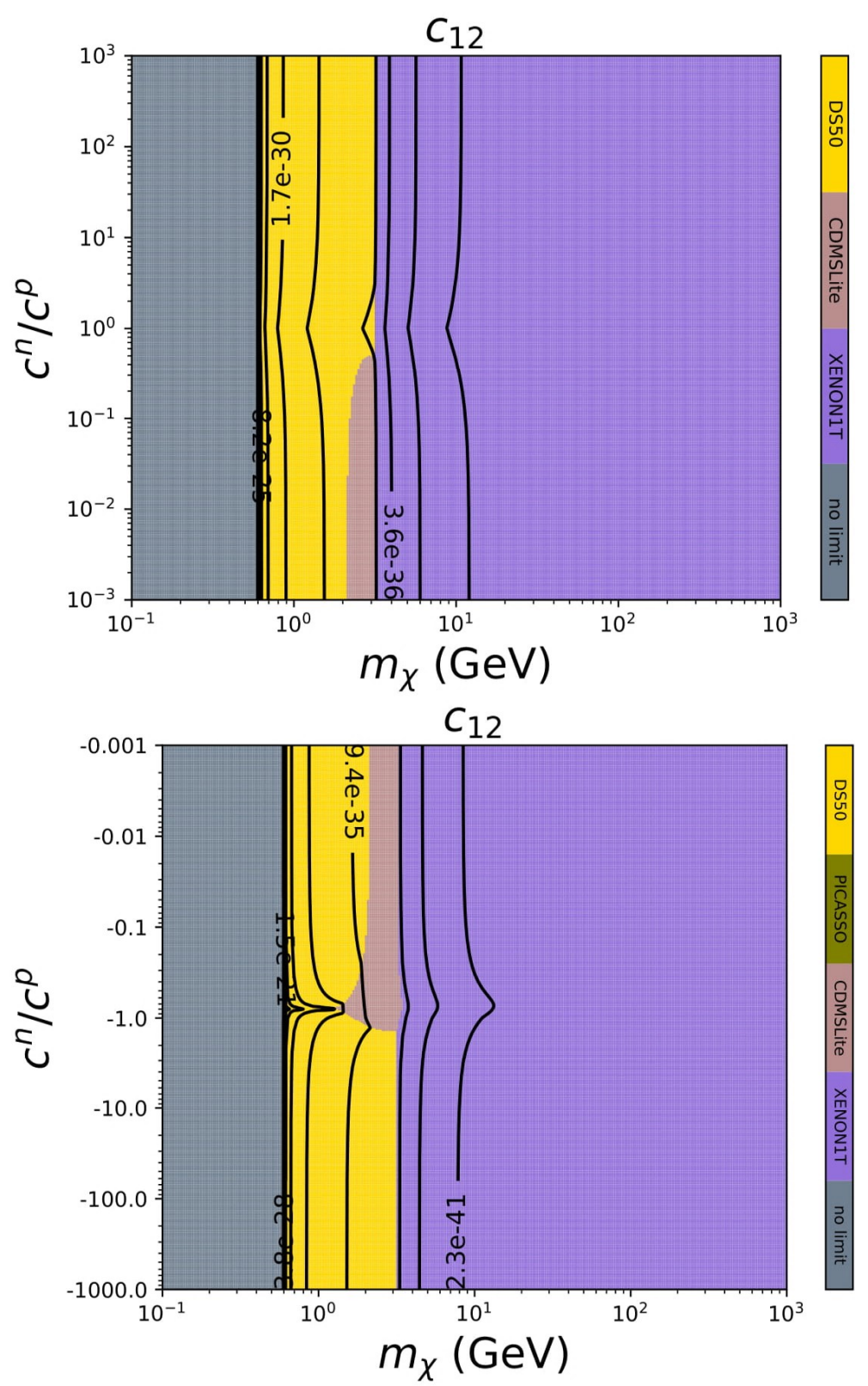

Figure 13: The same as in $\mathrm{Fig} 3$ for the operator $O_{12}$. 

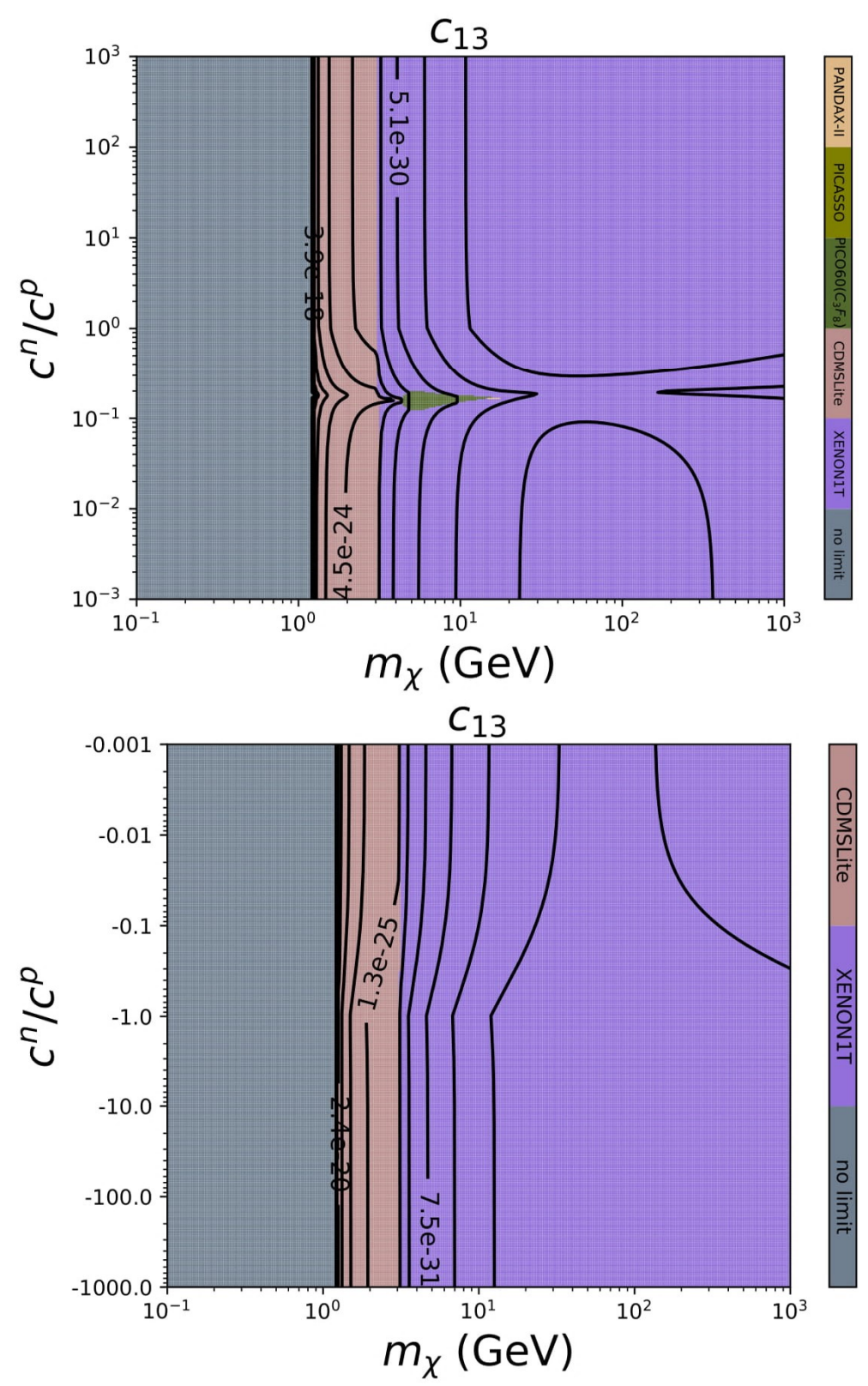

Figure 14: The same as in $\mathrm{Fig} 3$ for the operator $O_{13}$. 

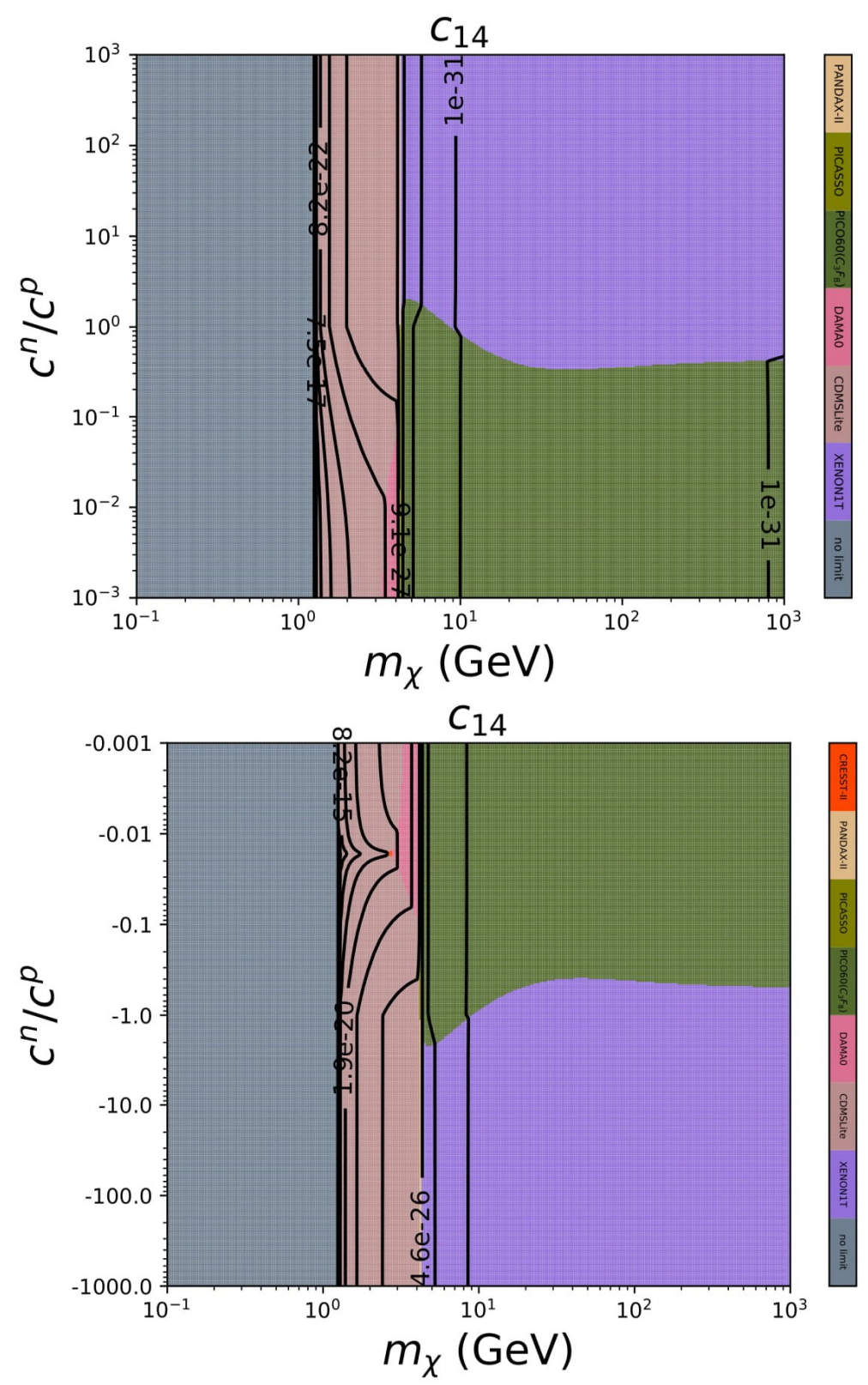

Figure 15: The same as in $\mathrm{Fig} 3$ for the operator $O_{14}$. 

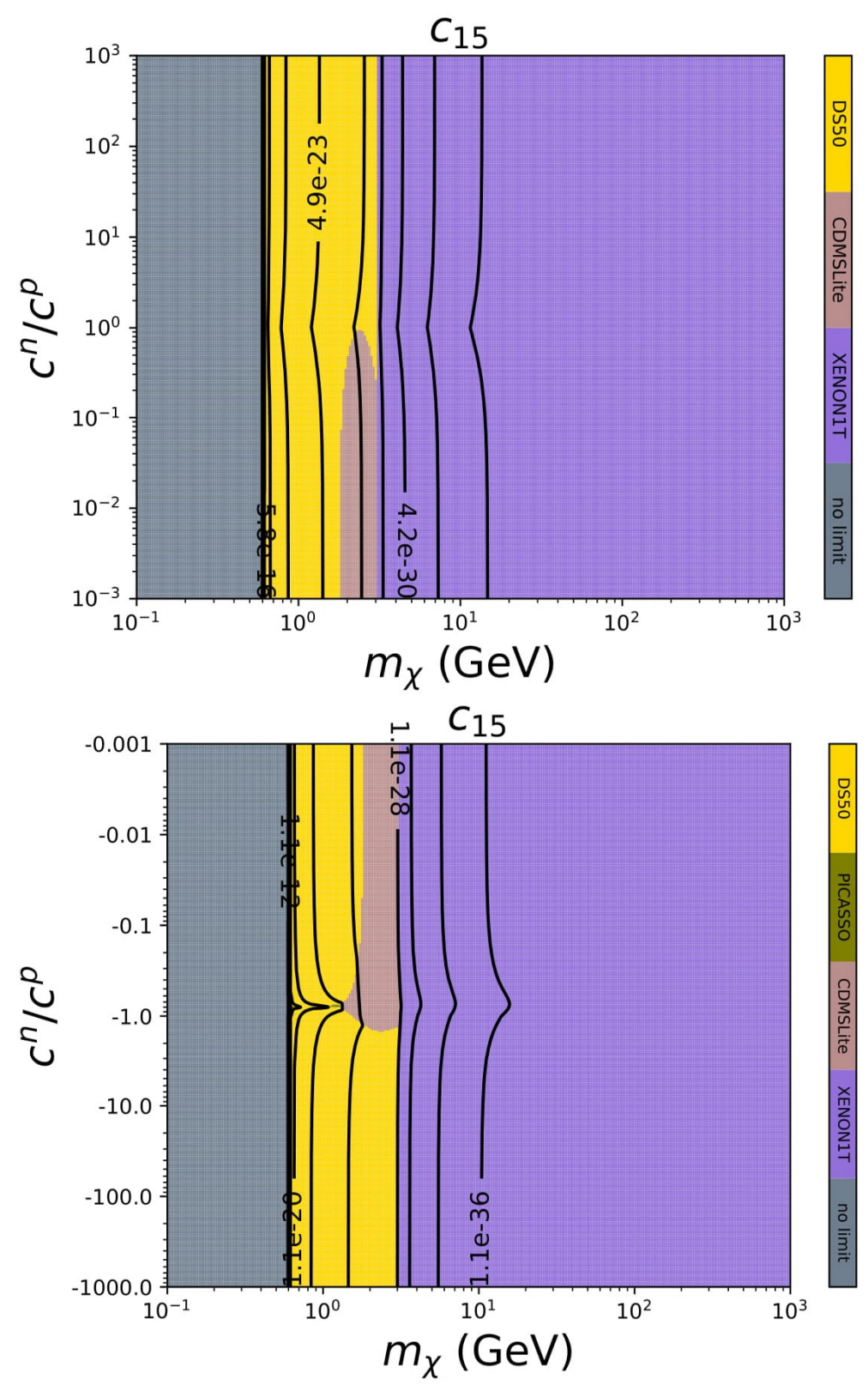

Figure 16: The same as in $\mathrm{Fig} 3$ for the operator $O_{15}$. 

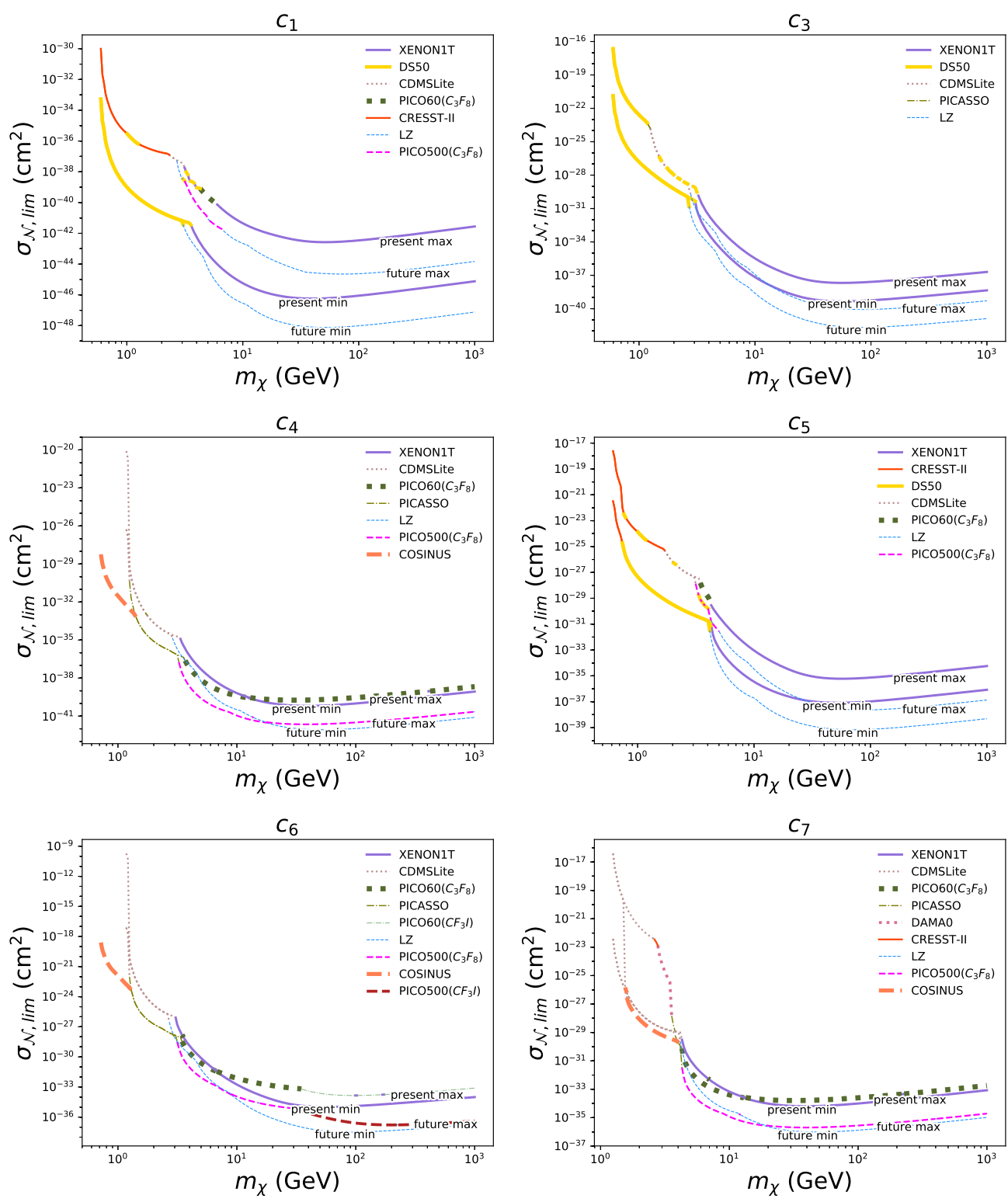

Figure 17: Most stringent bound among those from the experiments listed in Fig 1 on the effective WIMP-nucleon cross section $\sigma_{\mathcal{N}, \text { lim }}$ defined in Eq. (18) as a function of the WIMP mass $m_{\chi}$ for operators $c_{1}, c_{3}, c_{4}, c_{5}, c_{6}$ and $c_{7}$. In each plot the two curves indicated by "present min" and "present max" show the range of the limit from present experiments on $\sigma_{\mathcal{N}, \text { lim }}$ when $c^{n} / c^{p}$ is varied, while the curves indicated by "future min" and "future max" show the same range when the limits from projected experiments are included. In each curve the different styles indicate the experiment providing the most stringent bound, as shown by the legend. 

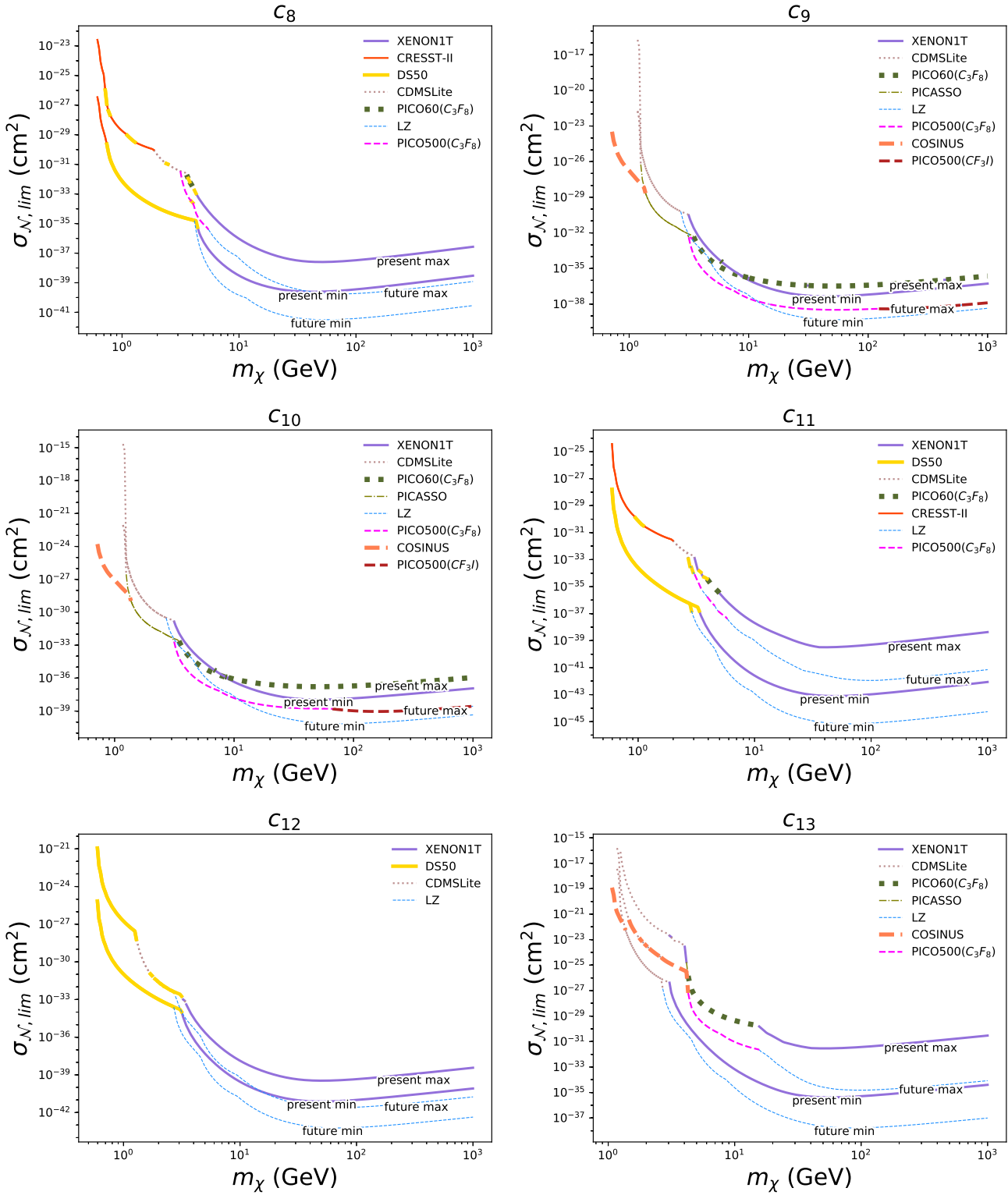

Figure 18: The same as in Fig. 17for operators $c_{8}, c_{9}, c_{10}, c_{11}, c_{12}$ and $c_{13}$. 

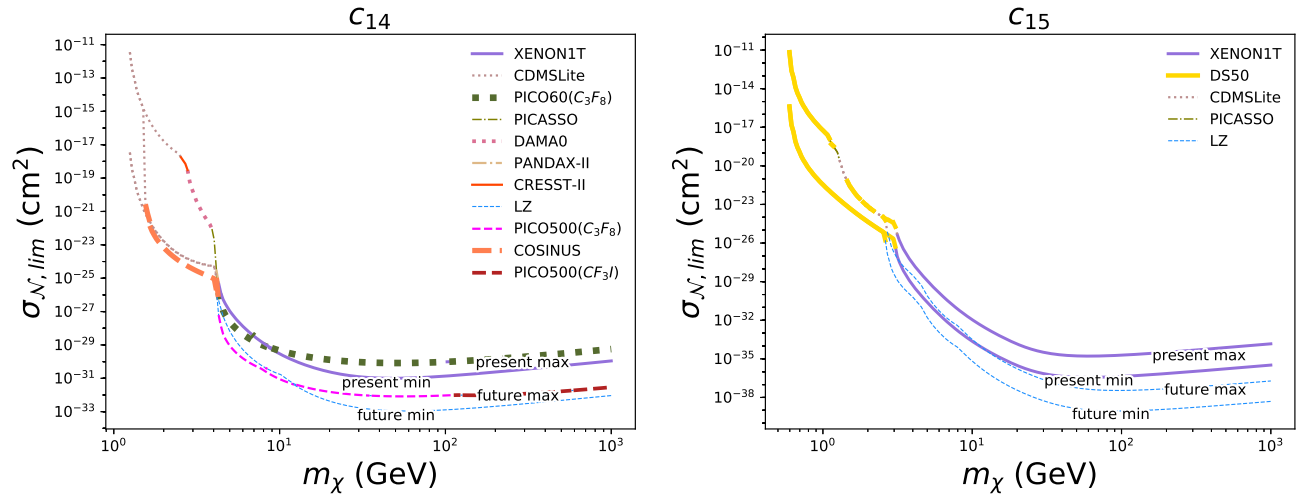

Figure 19: The same as in Fig. 18 for operators $c_{14}$ and $c_{15}$.

\section{Operator mixing}

In this Section we show how the results of the previous Section can be used to estimate approximate limits also in the case of more than one NR operator. To be quantitative, we consider here the specific examples of two interaction Lagrangians between the DM particle and quarks, valid at the scale $\Lambda=2 \mathrm{GeV}$. In the notation of [35]:

$$
Q_{2}^{(6)}=\sum_{q} \hat{C}_{2, q}^{(6)}\left(\bar{\chi} \gamma_{\mu} \gamma_{5} \chi\right)\left(\bar{q} \gamma^{\mu} q\right), \quad Q_{3}^{(6)}=\sum_{q} \hat{C}_{3, q}^{(6)}\left(\bar{\chi} \gamma_{\mu} \chi\right)\left(\bar{q} \gamma^{\mu} \gamma_{5} q\right)
$$

For simplicity, we assume in the following that the couplings are same for all quarks, $\hat{C}_{2, q}^{(6)}=\hat{C}_{2}^{(6)}$, $\hat{C}_{3, q}^{(6)}=\hat{C}_{3}^{(6)}$. With this assumption the non-relativistic limits of 19 are given by:

$$
\begin{aligned}
& Q_{2}^{(6)} \rightarrow c_{8} O_{8}+c_{9} O_{9}, \\
& Q_{3}^{(6)} \rightarrow c_{7} O_{7}+c_{9}^{\prime} O_{9},
\end{aligned}
$$

with $c_{8}=c_{8}^{n}=c_{8}^{p}=k_{8} \hat{C}_{2}^{(6)}, c_{9}=c_{9}^{n}=c_{9}^{p}=k_{9} \hat{C}_{2}^{(6)}, c_{7}=c_{7}^{n}=c_{7}^{p}=k_{7} \hat{C}_{3}^{(6)}, c_{9}^{\prime}=c_{9}^{\prime n}=c_{9}^{\prime p}=k_{9}^{\prime} \hat{C}_{3}^{(6)}$, with $k_{8}=6, k_{9}=4.89, k_{7}=-6$ and $k_{9}^{\prime}=6 m_{N} / m_{\chi}$ [35]. The corresponding calculation of the $90 \%$ C.L. upper bounds on the effective cross sections $\sigma_{2}^{(6)}=\left[\hat{\mathcal{C}}_{2}^{(6)}\right]^{2} \mu_{\chi \mathcal{N}}^{2} / \pi$ and $\sigma_{3}^{(6)}=\left[\hat{\mathcal{C}}_{3}^{(6)}\right]^{2} \mu_{\chi \mathcal{N}}^{2} / \pi$ are shown in Fig. 20 In each plot the different markers show which operator drives the constraint, $O_{i}<O_{j}$ meaning that the bound using only the contribution of $O_{i}$ is at least a factor of 3 better than the one obtained including only $O_{j}$. From such Figure one can see that, depending on the value of the WIMP mass, the constraints of different experiments can either be driven by the same NR operator or by different ones. In any case the result of the full calculation in Fig. 20 can be obtained with sufficient accuracy by using the NR results of Section 3. Let's assume the value $m_{\chi}=30 \mathrm{GeV}$ (at larger masses all limits for $C_{2}^{(6)}$ being driven by $O_{8}$ and those for $C_{3}^{(6)}$ by $O_{7}$ ). To proceed, for a given value of the WIMP mass one needs to obtain the corresponding bound on each NR coupling from the contour plots in the $m_{\chi}-r$ planes of Figs. 3 16 In particular the relativistic theory predicts for each NR coupling the $r_{i}=c^{n} / c^{p}$ ratio (in our simple example they are both equal to one). The rate on experiment $\exp$ can be written as: 
Table 2: Most stringent constraints on the effective cross section $\sigma_{\mathcal{N}, \text { lim }}$ for each of the couplings in the effective Hamiltonian of Eq. 3 among the present and future experiments included in our analysis. In each case the ratio $c^{n} / c^{p}$ is fixed to the value that corresponds to the most stringent bound.

\begin{tabular}{ccccc}
\hline Coupling & \multicolumn{2}{c}{ Present } & \multicolumn{2}{c}{ Future } \\
\hline & $m_{\chi}(\mathrm{GeV})$ & $\sigma_{\mathcal{N}, \text { lim }}\left(\mathrm{cm}^{2}\right)$ & $m_{\chi}(\mathrm{GeV})$ & $\sigma_{\mathcal{N}, \text { lim }}\left(\mathrm{cm}^{2}\right)$ \\
$c_{1}$ & 40.6 & $5.7 \times 10^{-47}$ & 52.0 & $7.6 \times 10^{-49}$ \\
$c_{3}$ & 57.0 & $4.5 \times 10^{-40}$ & 82.5 & $2.1 \times 10^{-42}$ \\
$c_{4}$ & 39.4 & $6.3 \times 10^{-41}$ & 52.0 & $8.4 \times 10^{-43}$ \\
$c_{5}$ & 52.0 & $8.2 \times 10^{-38}$ & 70.7 & $6.9 \times 10^{-40}$ \\
$c_{6}$ & 57.0 & $1.0 \times 10^{-35}$ & 90.5 & $3.3 \times 10^{-38}$ \\
$c_{7}$ & 39.4 & $6.4 \times 10^{-35}$ & 47.4 & $9.8 \times 10^{-37}$ \\
$c_{8}$ & 43.2 & $2.4 \times 10^{-40}$ & 55.3 & $3.2 \times 10^{-42}$ \\
$c_{9}$ & 45.9 & $4.5 \times 10^{-38}$ & 57.0 & $4.9 \times 10^{-40}$ \\
$c_{10}$ & 52.0 & $1.1 \times 10^{-38}$ & 77.6 & $6.9 \times 10^{-41}$ \\
$c_{11}$ & 48.9 & $7.8 \times 10^{-44}$ & 64.5 & $6.9 \times 10^{-46}$ \\
$c_{12}$ & 50.4 & $7.6 \times 10^{-42}$ & 68.6 & $5.9 \times 10^{-44}$ \\
$c_{13}$ & 57.0 & $4.2 \times 10^{-36}$ & 85.1 & $1.6 \times 10^{-38}$ \\
$c_{14}$ & 47.4 & $1.0 \times 10^{-31}$ & 58.8 & $1.1 \times 10^{-33}$ \\
$c_{15}$ & 60.6 & $3.6 \times 10^{-37}$ & 93.3 & $8.8 \times 10^{-40}$ \\
\hline
\end{tabular}

Table 3: Most stringent constraints on the effective cross section $\sigma_{\mathcal{N}, l i m}$ for each of the couplings in the effective Hamiltonian of Eq. 3 among the present and future experiments included in our analysis. In each case the ratio $c^{n} / c^{p}$ is fixed to the value that corresponds to the less stringent bound.

\begin{tabular}{ccccc}
\hline Coupling & \multicolumn{2}{c}{ Present } & \multicolumn{2}{c}{ Future } \\
\hline & $m_{\chi}(\mathrm{GeV})$ & $\sigma_{\mathcal{N}, \text { lim }}\left(\mathrm{cm}^{2}\right)$ & $m_{\chi}(\mathrm{GeV})$ & $\sigma_{\mathcal{N}, \text { lim }}\left(\mathrm{cm}^{2}\right)$ \\
$c_{1}$ & 52.0 & $2.7 \times 10^{-43}$ & 72.9 & $2.2 \times 10^{-45}$ \\
$c_{3}$ & 57.0 & $2.1 \times 10^{-38}$ & 85.1 & $8.4 \times 10^{-41}$ \\
$c_{4}$ & 29.8 & $1.8 \times 10^{-40}$ & 37.0 & $2.2 \times 10^{-42}$ \\
$c_{5}$ & 57.0 & $6.0 \times 10^{-36}$ & 87.7 & $2.3 \times 10^{-38}$ \\
$c_{6}$ & 96.2 & $1.4 \times 10^{-34}$ & 207.8 & $1.7 \times 10^{-37}$ \\
$c_{7}$ & 31.7 & $1.6 \times 10^{-34}$ & 39.4 & $2.0 \times 10^{-36}$ \\
$c_{8}$ & 50.4 & $2.5 \times 10^{-38}$ & 72.9 & $1.8 \times 10^{-40}$ \\
$c_{9}$ & 50.4 & $3.3 \times 10^{-37}$ & 53.6 & $3.3 \times 10^{-39}$ \\
$c_{10}$ & 50.4 & $1.7 \times 10^{-37}$ & 162.4 & $9.2 \times 10^{-40}$ \\
$c_{11}$ & 39.4 & $3.2 \times 10^{-40}$ & 99.2 & $1.1 \times 10^{-42}$ \\
$c_{12}$ & 50.4 & $3.4 \times 10^{-40}$ & 72.9 & $2.5 \times 10^{-42}$ \\
$c_{13}$ & 52.0 & $2.9 \times 10^{-32}$ & 99.2 & $1.5 \times 10^{-35}$ \\
$c_{14}$ & 52.0 & $8.2 \times 10^{-31}$ & 55.3 & $8.2 \times 10^{-33}$ \\
$c_{15}$ & 60.6 & $1.6 \times 10^{-35}$ & 93.3 & $3.5 \times 10^{-38}$ \\
\hline
\end{tabular}



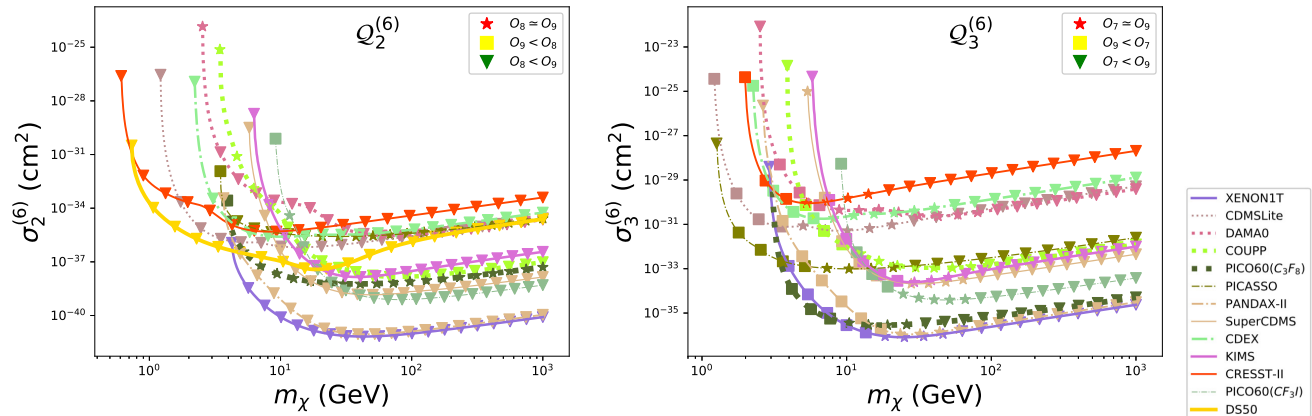

Figure 20: Current 90\% C.L. exclusion plots to the effective cross sections $\sigma_{2}^{(6)}=\left[\hat{\mathcal{C}}_{2}^{(6)}\right]^{2} \mu_{\chi N}^{2} / \pi$ and $\sigma_{3}^{(6)}=\left[\hat{C}_{3}^{(6)}\right]^{2} \mu_{\chi N}^{2} / \pi$. Different markers show which operator drives the limit, $O_{i}<O_{j}$ meaning that the bound using only the contribution of $O_{i}$ is at least a factor of 3 better than the one obtained including only $O_{j}$.

$$
R_{\text {exp }}=\left(\hat{C}_{a}^{(d)}\right)^{2} \sum_{i} k_{i}^{2} \mathcal{R}_{i, \exp }\left(m_{\chi}, r_{i}\right)=\sum_{i} c_{i}^{2} \mathcal{R}_{i, \exp }\left(m_{\chi}, r_{i}\right)
$$

where the sum over operators $i$ neglects interferences and $\mathcal{R}_{i, \exp }$ is a response function that depends on the experimental details. Assuming no cancellations in the sum the bound on the coupling $\hat{C}_{a}^{(d)}$ from the upper bound $R_{\text {explim }}$ on the count rate can be approximated by:

$$
\left(\hat{\mathcal{C}}_{a}^{(d)}\right)^{2} \lesssim \min _{i, \exp } \frac{R_{\text {exp }, \text { lim }}}{k_{i}^{2} \mathcal{R}_{i, \exp }\left(m_{\chi}, r_{i}\right)}=\min _{i} \frac{1}{k_{i}^{2}} \min _{\exp } \frac{R_{\text {exp }, \text { lim }}}{\mathcal{R}_{i, \exp }\left(m_{\chi}, r_{i}\right)}=\min _{i} \frac{1}{k_{i}^{2}}\left[c_{i}^{\lim }\left(m_{\chi}, r_{i}\right)\right]^{2} .
$$

The constraint:

$$
\left[c_{i}^{\lim }\left(m_{\chi}, r_{i}\right)\right]^{2}=\min _{\exp } \frac{R_{\text {exp }, \text { lim }}}{\mathcal{R}_{i, \exp }\left(m_{\chi}, r_{i}\right)}
$$

can be read from the planes of Figs. 3 16. Notice that this procedure does not require that the same operator dominates at the same WIMP mass in different experiments, because the $k_{i}$ quantities depend on the interaction but not on the experiment.

In our specific example, for $m_{\chi} \simeq 30 \mathrm{GeV}, r=1$ the bound for the operator $O_{7}$ can be obtained from Fig. 8 ( $\left(\sigma_{\text {lim }}^{7} \simeq 6.9 \times 10^{-35} \mathrm{~cm}^{2}\right)$, that for the operator $O_{8}$ can be obtained from Fig. $9\left(\sigma_{\text {lim }}^{8} \simeq\right.$ $\left.2.7 \times 10^{-40} \mathrm{~cm}^{2}\right)$ and that for the operator $O_{9}$ can be obtained from Fig. $10\left(\sigma_{\text {lim }}^{9} \simeq 5.5 \times 10^{-38}\right.$ $\left.\mathrm{cm}^{2}\right)$. Then assuming dominance of one coupling at a time one gets for model $Q_{2}^{(6)}$ either $k_{8}^{2} \sigma_{2}^{(6)}<$ $\sigma_{\text {lim }}^{8}$ or $k_{9}^{2} \sigma_{2}^{(6)}<\sigma_{\text {lim }}^{9}$. The most constraining of the two bounds is $\sigma_{2}^{(6)} \simeq \sigma_{\text {lim }}^{8} / k_{8}^{2} \simeq 7.5 \times 10^{-42}$ $\mathrm{cm}^{2}$, in agreement to the result in Fig. 20. Proceeding in the same way, for model $Q_{3}^{(6)}$ either $k_{7}^{2} \sigma_{3}^{(6)}<\sigma_{\text {lim }}^{7}$ or $\left(k_{9}^{\prime}\right)^{2} \sigma_{3}^{(6)}<\sigma_{\text {lim }}^{9}$. In this case the two bounds are similar, $\sigma_{3}^{(6)} \simeq \sigma_{\text {lim }}^{7} / k_{7}^{2} \simeq$ $1.9 \times 10^{-36} \mathrm{~cm}^{2}, \sigma_{3}^{(6)} \simeq \sigma_{\text {lim }}^{9} /\left(k_{9}^{\prime}\right)^{2} \simeq 1.6 \times 10^{-36} \mathrm{~cm}^{2}$. Since in this case the two operators have similar contributions this approximate bound is about a factor of 2 weaker than the result in Fig. 20. $\sigma_{3}^{(6)} \lesssim 8.4 \times 10^{-37} \mathrm{~cm}^{2}$.

Although in the present analysis we only considered the case of a contact interaction, a similar procedure can be generalized in a straightforward way to the case of relativistic couplings whose NR limit leads to operators multiplied by a propagator $q^{-2}$. For instance, the coupling 
$1 / q^{2} \bar{\chi} \sigma^{\mu \nu} \frac{q_{v}}{m_{N}} \chi \bar{q} \gamma_{\mu} q$ (a DM particle coupling through a magnetic dipole moment) leads to the combination $-\frac{1}{2 m_{X}} O_{1}+\frac{2 m_{N}}{q^{2}} O_{5}-2 m_{N}\left(\frac{1}{m_{N}^{2}} O_{4}-\frac{1}{q^{2}} O_{6}\right)$. This would require to extend our systematic discussion in Figs 316 to the limits for the generalized operator $q^{-2} O_{i}$ and $q^{-4} O_{i}$.

\section{Conclusions}

Assuming for WIMPs a Maxwellian velocity distribution in the Galaxy we have explored in a systematic way the relative sensitivity of an extensive set of 14 existing and 4 projected Dark Matter direct detection experiments to each of the couplings that parameterize the most general non-relativistic effective Hamiltonian allowed by Galilean invariance for a contact interaction driving the elastic scattering off nuclei of WIMPs of spin $1 / 2$. We have performed our analysis in terms of two free parameters: the WIMP mass and the ratio between the WIMP-neutron and the WIMP-proton couplings $c^{n} / c^{p}$. For each coupling we have provided contour plots in the $m_{\chi}-c^{n} / c^{p}$ plane of the most stringent $90 \%$ C.L. bound on the WIMP-nucleon cross section and indicated with different shadings the experiment providing the most constraining bound. In Appendix D we will also introduce NRDD_constraints, a simple interpolating code written in Python that allows to extract the numerical value of the bound as a function of the WIMP mass $m_{\chi}$ and of the coupling ratio $c^{n} / c^{p}$ for each NR coupling.

We found that 9 present experiments out of the total of 14 considered in the present analysis provide the most stringent bound on some of the effective couplings for a given choice of $\left(m_{\chi}, c^{n} / c^{p}\right)$ : this is evidence of the complementarity of different target nuclei and/or different combinations of count-rates and energy thresholds when the search of a DM particle is extended to a wide range of possible interactions.

In particular in our analysis we have adopted the approach of taking all published result "at face value", and refrained from discussing their relative robustness. It is however worth stressing out here that, while the DarkSide 50 constraint appears from our analysis to be quite competitive at low WIMP masses compared to other experiments, it makes use of an ionization yield that has not been measured below $10 \mathrm{keV}$ (see Appendix B.2.

In our analysis the lower part of the 2-sigma DAMA modulation amplitude region in the $m_{\chi}-$ $\sigma_{p}$ plane is included as if it were an additional constraint, in order to locate in the parameter space possible regions where the DAMA excess is compatible to other constraints. DAMA does not appear as the most constraining bound in any of the figures $3-16$, indicating that an explanation of its annual modulation excess in terms of a WIMP signals is in tension with the constraints of other experiments no matter which of the effective operators among those in Eq.(3) is assumed to dominate in the WIMP-nucleus interaction. This result is in agreement with the findings of Ref. [44]. While in our analysis we assumed dominance of one NR operator at a time, we have shown how our results can be used to estimate approximate limits also in the case of interactions which depend on more than one NR operator.

\section{Acknowledgements}

This research was supported by the Basic Science Research Program through the National Research Foundation of Korea (NRF) funded by the Ministry of Education, grant number 2016R1D1A1A09917964. 


\section{Appendix A. WIMP response functions}

We collect here the WIMP particle-physics response functions introduced in Eq.12 and adapted from [25, 26]:

$$
\begin{aligned}
R_{M}^{\tau \tau^{\prime}}\left(v_{T}^{\perp 2}, \frac{q^{2}}{m_{N}^{2}}\right)= & c_{1}^{\tau} c_{1}^{\tau^{\prime}}+\frac{j_{\chi}\left(j_{\chi}+1\right)}{3}\left[\frac{q^{2}}{m_{N}^{2}} v_{T}^{\perp 2} c_{5}^{\tau} c_{5}^{\tau^{\prime}}+v_{T}^{\perp 2} c_{8}^{\tau} c_{8}^{\tau^{\prime}}+\frac{q^{2}}{m_{N}^{2}} c_{11}^{\tau} c_{11}^{\tau^{\prime}}\right] \\
R_{\Phi^{\prime \prime}}^{\tau \tau^{\prime}}\left(v_{T}^{\perp 2}, \frac{q^{2}}{m_{N}^{2}}\right)= & {\left[\frac{q^{2}}{4 m_{N}^{2}} c_{3}^{\tau} c_{3}^{\tau^{\prime}}+\frac{j_{\chi}\left(j_{\chi}+1\right)}{12}\left(c_{12}^{\tau}-\frac{q^{2}}{m_{N}^{2}} c_{15}^{\tau}\right)\left(c_{12}^{\tau^{\prime}}-\frac{q^{2}}{m_{N}^{2}} c_{15}^{\tau^{\prime}}\right)\right] \frac{q^{2}}{m_{N}^{2}} } \\
R_{\Phi^{\prime \prime} M}^{\tau \tau^{\prime}}\left(v_{T}^{\perp 2}, \frac{q^{2}}{m_{N}^{2}}\right)= & {\left[c_{3}^{\tau} c_{1}^{\tau^{\prime}}+\frac{j_{\chi}\left(j_{\chi}+1\right)}{3}\left(c_{12}^{\tau}-\frac{q^{2}}{m_{N}^{2}} c_{15}^{\tau}\right) c_{11}^{\tau^{\prime}}\right] \frac{q^{2}}{m_{N}^{2}} } \\
R_{\tilde{\Phi}^{\prime}}^{\tau \tau^{\prime}}\left(v_{T}^{\perp 2}, \frac{q^{2}}{m_{N}^{2}}\right)= & {\left[\frac{j_{\chi}\left(j_{\chi}+1\right)}{12}\left(c_{12}^{\tau} c_{12}^{\tau^{\prime}}+\frac{q^{2}}{m_{N}^{2}} c_{13}^{\tau} c_{13}^{\tau^{\prime}}\right)\right] \frac{q^{2}}{m_{N}^{2}}, } \\
R_{\Sigma^{\prime \prime}}^{\tau \tau^{\prime}}\left(v_{T}^{\perp 2}, \frac{q^{2}}{m_{N}^{2}}\right)= & \frac{q^{2}}{4 m_{N}^{2}} c_{10}^{\tau} c_{10}^{\tau^{\prime}}+\frac{j_{\chi}\left(j_{\chi}+1\right)}{12}\left[c_{4}^{\tau} c_{4}^{\tau^{\prime}}+\right. \\
R_{\Sigma^{\prime}}^{\tau \tau^{\prime}}\left(v_{T}^{\perp 2}, \frac{q^{2}}{m_{N}^{2}}\right)= & \left.\frac{q^{2}}{m_{N}^{2}}\left(c_{4}^{\tau} c_{6}^{\tau^{\prime}}+c_{6}^{\tau} c_{4}^{\tau^{\prime}}\right)+\frac{q^{4}}{m_{N}^{4}} c_{6}^{\tau} c_{6}^{\tau^{\prime}}+v_{T}^{\perp 2} c_{12}^{\tau} c_{12}^{\tau^{\prime}}+\frac{q^{2}}{m_{N}^{2}} v_{T}^{\perp 2} c_{13}^{\tau} c_{13}^{\tau^{\prime}} c_{3}^{\tau} c_{3}^{\tau^{\prime}}+v_{T}^{\perp 2} c_{7}^{\tau} c_{7}^{\tau^{\prime}}\right]+\frac{j_{\chi}\left(j_{\chi}+1\right)}{12}\left[c_{4}^{\tau} c_{4}^{\tau^{\prime}}+\right. \\
R_{\Delta \Sigma^{\prime}}^{\tau \tau^{\prime}}\left(v_{T}^{\perp 2}, \frac{q^{2}}{m_{N}^{2}}\right)= & \left.\frac{q^{2}}{m_{N}^{2}} c_{9}^{\tau} c_{9}^{\tau^{\prime}}+\frac{v_{T}^{\perp 2}}{2}\left(c_{12}^{\tau}-\frac{q^{2}}{m_{N}^{2}} c_{15}^{\tau}\right)\left(c_{12}^{\tau^{\prime}}-\frac{q^{2}}{m_{N}^{2}} c_{15}^{\tau \prime}\right)+\frac{q^{2}}{2 m_{N}^{2}} v_{T}^{\perp 2} c_{14}^{\tau} c_{14}^{\tau^{\prime}}\right] \\
R_{\Delta}^{\tau \tau^{\prime}}\left(v_{T}^{\perp 2}, \frac{q^{2}}{m_{N}^{2}}\right)= & \frac{j_{\chi}\left(j_{\chi}+1\right)}{3}\left(\frac{q^{2}}{m_{N}^{2}} c_{5}^{\tau} c_{5}^{\tau^{\prime}}+c_{8}^{\tau} c_{8}^{\tau^{\prime}}\right) \frac{q^{2}}{m_{N}^{2}}, \\
m_{5} & \left.c_{5}^{\tau} c_{4}^{\tau^{\prime}}-c_{8}^{\tau} c_{9}^{\tau^{\prime}}\right) \frac{q^{2}}{m_{N}^{2}} .
\end{aligned}
$$

\section{Appendix B. Experiments}

In the present analysis we include an extensive set of constraints that are representative of the different techniques used to search for DM: XENON1T [6], PANDAX-II [7], KIMS [8], CDMSlite [9], SuperCDMS [10], COUPP [11], PICASSO [12], PICO-60 (using a $C F_{3} I$ target [13] and a $C_{3} F_{8}$ one [14]) CRESST-II [15, 30], DAMA (modulation data [2, 3, 4, 5] and average count rate [31]), CDEX [16] and DarkSide-50 [18]. We also consider projected sensitivities of some future detectors: LZ [20], PICO-500 [32] and COSINUS [33]. In the following, if not specified otherwise we adopt for the energy resolution a Gaussian form, $\mathcal{G}\left(E^{\prime}, E_{e e}\right)=\operatorname{Gauss}\left(E^{\prime} \mid E_{e e}, \sigma\right)=$ $1 /(\sqrt{2 \pi} \sigma) \exp \left(-\left(E^{\prime}-E_{e e}\right) / 2 \sigma^{2}\right)$. The quenching factor of bolometers (SuperCDMS, CRESST-II, COSINUS) is assumed to be equal to 1 .

\section{Appendix B.1. Xenon: XENON1T, PANDAX-II and LZ}

For XENON1T we have assumed 7 WIMP candidate events in the range of $3 \mathrm{PE} \leq S_{1} \leq 70 \mathrm{PE}$, as shown in Fig. 3 of Ref. [6] for the primary scintillation signal S1 (directly in Photo Electrons, $\mathrm{PE}$ ), with an exposure of 278.8 days and a fiducial volume of 1.3 ton of xenon. We have used the 
efficiency taken from Fig. 1 of [6] and employed a light collection efficiency $g_{1}=0.055$; for the light yield $L_{y}$ we have extracted the best estimation curve for photon yields $\left\langle n_{p h}\right\rangle / E$ from Fig. 7 in [47] with an electric field of $90 \mathrm{~V} / \mathrm{cm}$.

On the other hand for PANDAX-II we implemented the combined result of Run 9 and Run 10 with $\simeq 0.2$ events after background subtraction in the range $3 \mathrm{PE} \leq S_{1} \leq 45 \mathrm{PE}$ in the lower half of the signal band, as shown in Fig.4, for a total exposure of 79.6+77.1 days and a fiducial mass of $361.5 \mathrm{~kg}$ [7]. We have taken the efficiency from Fig.16 and $L_{y}$ from Fig.13b of the supplemental material provided in [7]. To reproduce the published PANDAX-II combined Run 9 and Run 10 result we adopted a photon gain $g_{1}=0.0557$.

LUX-ZEPLIN (LZ) is a next generation dual-phase xenon DM direct detection experiment which will operate with an active mass of 7 tonnes. We assumed an exposure of $5.6 \times 10^{6} \mathrm{~kg}$ days [20]. Its sensitivity to low mass WIMPs will depend strongly on the low energy nuclear recoil efficiency. To obtain projections in Ref. [20] an extrapolation down to $0.1 \mathrm{keV}$ following Lindhard theory is used. Lacking any direct measurement of this quantity at low energy we use the light yield of Fig.2 of [20] with a hard cutoff at $1.1 \mathrm{keV}$. We assume no signal in the lower half nuclear recoil band below the red curve of Fig.7 in [20] and a neutrino background of 12 events for $S_{1}<4 \mathrm{PE}$.

For XENON1T, PANDAX-II and LZ experiments we have modeled the energy resolution combining a Poisson fluctuation of the observed primary signal $S_{1}$ compared to $\left\langle S_{1}\right\rangle$ and a Gaussian response of the photomultiplier with $\sigma_{P M T}=0.5$, so that:

$$
\mathcal{G}_{X e}\left(E_{R}, S\right)=\sum_{n=1}^{\infty} \operatorname{Gauss}\left(S \mid n, \sqrt{n} \sigma_{P M T}\right) \operatorname{Poiss}\left(n,<S\left(E_{R}\right)>\right),
$$

with $\operatorname{Poiss}(n, \lambda)=\lambda^{n} / n ! \exp (-\lambda)$.

\section{Appendix B.2. Argon: DarkSide-50}

The analysis of DarkSide-50 [18] is based on the ionization signal extracted from liquid argon with an exposure of $6786.0 \mathrm{~kg}$ days. The measured spectrum for $N_{e^{-}}<50$ (with $N_{e^{-}}$the number of extracted electrons) is shown in Fig. 7 of [18], and shows an excess for $4<N_{e^{-}}<7 N_{e^{-}}$ compared to a simulation of the background components from known radioactive contaminants. Following Ref.[18] we have subtracted the background minimizing the likelihood function:

$$
-2 \mathcal{L}=\sum_{i} \frac{\left(\sigma S_{i}+\rho b_{i}-x_{i}\right)^{2}}{\sigma_{i}^{2}}
$$

where $i$ represents the energy bin, $x_{i}$ the measured spectrum with error $\sigma_{i}$, while $\sigma S_{i}$ and $\rho b_{i}$ are the DM signal and the background, respectively, with $\sigma$ and $\rho$ arbitrary normalization factors ( $\sigma$ is identified with the effective WIMP-proton cross section $\sigma_{p}$ ). In particular we obtain the $90 \%$ C.L. upper bound on $\sigma_{p}$ by taking its profile likelihood with $-2 \mathcal{L}-[-2 \mathcal{L}]_{\min }=n^{2}$ and $n=1.28$. We take $x_{i}, \sigma_{i}$ and $b_{i}$ from Fig.7 of [18]. The ionization yield of argon has been measured only down to $\lesssim 10 \mathrm{keVnr}$, while DS50 uses a model fit to calibration data. We use the latter as taken from Fig. 6 of [18] with a hard cut at $0.15 \mathrm{keVnr}$, the lowest energy for which it is provided. We take the efficiency from Fig. 1 of [18]. The signal/background ratio in DS50 is at the percent level, so the background subtraction procedure is sensitive to the details of its implementation. Following the background subtraction procedure described above we reproduce fairly well the published DS50 exclusion plot with the exception of the mass range $4 \mathrm{GeV} \lesssim m_{\chi} \lesssim 15 \mathrm{GeV}$, 


\begin{tabular}{|c|c|c|}
\hline$E_{\text {th }}(\mathrm{keV})$ & Event rate (events/kg/day) & Fluctuation \\
\hline 1.0 & -1.5 & 3.8 \\
1.5 & -0.2 & 1.0 \\
2.7 & 0.3 & 0.8 \\
6.6 & -0.8 & 1.8 \\
15.7 & -1.4 & 2.3 \\
36.8 & 0.3 & 1.0 \\
\hline
\end{tabular}

Table B.4: Observed number of events and 1-sigma statistical fluctuations (extracted from Fig. 4 of Ref. [12]) for each operating threshold used in PICASSO.

where our procedure is less constraining for a SI interaction by a factor up to $\simeq 3.5$ compared to the published one. Given the large uncertainties involved we consider such result acceptable.

Appendix B.3. Germanium: SuperCDMS, CDMSlite and CDEX

The latest SuperCDMS analysis [10] observed 1 event between 4 and $100 \mathrm{keVnr}$ with an exposure of $1690 \mathrm{~kg}$ days. We have taken the efficiency from Fig.1 of [10] and the energy resolution $\sigma=\sqrt{0.293^{2}+0.056^{2} E_{e e}}$ from [48]. To analyze the observed spectrum we apply the optimal interval method [46].

For CDMSlite we considered the energy bin of $0.056 \mathrm{keV}<E^{\prime}<1.1 \mathrm{keV}$ with a measured count rate of $1.1 \pm 0.2\left[\mathrm{keV} \mathrm{kg} \mathrm{day}^{-1}\right.$ (Full Run 2 rate, Table II of Ref. [9]). We have taken the efficiency from Fig.4 of [9] and the energy resolution $\sigma=\sqrt{\sigma_{E}^{2}+B E_{R}+\left(A E_{R}\right)^{2}}$, with $\sigma_{E}=9.26$ $\mathrm{eV}, A=5.68 \times 10^{-3}$ and $B=0.64 \mathrm{eV}$ from Section IV.A of [9].

CDEX [16] uses a germanium target with an exposure of $737.1 \mathrm{~kg}$ days. We analyze the residual excess events for $160 \mathrm{eVee}<E^{\prime}<2.56 \mathrm{keVee}$ detected in the Anti-Compton Veto spectrum of Fig. 7 in [16] and the efficiency from Fig.4 of [16]. For the quenching factor of germanium we use the Lindhard formula [49]:

$$
Y\left(E_{R}\right)=\frac{k \cdot g(\epsilon)}{1+k \cdot g(\epsilon)},
$$

where $g(\epsilon)=3 \epsilon^{0.15}+0.7 \epsilon^{0.6}+\epsilon, \epsilon=11.5 E_{R}\left(\mathrm{keV}_{n r}\right) Z^{-7 / 3}$, and $Z$ the atomic number. For germanium, $k=0.157$ and $Z=32$.

\section{Appendix B.4. Fluorine: PICASSO, PICO-60 and PICO-500}

Bubble chambers are threshold experiments for which we employ the nucleation probability:

$$
\mathcal{P}_{T}\left(E_{R}\right)=1-\exp \left[-\alpha_{T} \frac{E_{R}-E_{t h}}{E_{t h}}\right] .
$$

The PICASSO experiment [12] uses $C_{4} F_{10}$ as a target and operated its runs with six energy thresholds. For each threshold we provide the corresponding number of observed events and statistical fluctuations in Table B.4 (extracted from Fig. 4 of Ref. [12]). For the nucleation probability we used Eq. (B.4) with $\alpha_{C}=\alpha_{F}=5$.

The target material of PICO-60 and PICO-500 is $C_{3} F_{8}$. For PICO-60 [14] only the threshold $E_{t h}=3.3 \mathrm{keV}$ is analyzed with a total exposure of $1167.0 \mathrm{~kg}$ days and no event detected. We have assumed the nucleation probability in Fig. 4 of [50]. 


\begin{tabular}{|c|c|c|}
\hline$E_{t h}(\mathrm{keV})$ & exposure (kg day) & measured events \\
\hline 7.8 & 55.8 & 2 \\
11 & 70 & 3 \\
15.5 & 311.7 & 8 \\
\hline
\end{tabular}

Table B.5: The operating thresholds with corresponding exposures and measured events for COUPP.

PICO-500 is a projected future extension of PICO-60 [32] with 250 liters of fiducial volume. We have assumed an exposure of 6 months at the energy threshold $E_{t h}=3.2 \mathrm{keV}$ and an exposure of one year at the energy threshold $E_{t h}=10 \mathrm{keV}$. We assumed no candidate events, and the same nucleation probabilities of PICO-60.

\section{Appendix B.5. Fluorine+Iodine: COUPP, PICO-60 and PICO-500}

COUPP is bubble chamber using a $C F_{3} I$ target. For each operating threshold used in COUPP the corresponding exposure and number of measured events are summarized in Table B.5. For fluorine and carbon we use the nucleation probability of Eq. B.4 with $\alpha=0.15$. For iodine we adopt instead a step function with nucleation probability equal to 1 above the energy threshold.

PICO-60 can also employ a $C F_{3} I$ target. For the analysis of Ref.[13] we adopt an energy threshold of $13.6 \mathrm{keV}$ and an exposure of $1335 \mathrm{~kg}$ days. The nucleation probabilities for each target element are taken from Fig.4 in [13].

PICO-500 is also planned to use a $C F_{3} I$ target [32]. As in the case of $C_{3} F_{8}$ we adopt an exposure of 6 months for $E_{t h}=3 \mathrm{keV}$ and of one year for $E_{t h}=10 \mathrm{keV}$, with no candidate events. We have taken the nucleation probabilities from Fig.4 of [13].

\section{Appendix B.6. Sodium Iodide: DAMA, KIMS and COSINUS}

For DAMA we consider both the upper bound from the average count rate (DAMA0) and the latest result for the annual modulation amplitudes. In particular we include the lower part of the 2-sigma modulation amplitude region in the $m_{\chi}-\sigma_{p}$ plane in the analysis of the most stringent bound of Section 3 as if it were an additional constraint, in order to locate possible regions of compatibility between the DAMA excess and other constraints in the parameter space. For DAMA0 we have taken the average count rates from [31] (rebinned from 0.25-keVee- to 0.5-keVee-width bins) from $2 \mathrm{keVee}$ to $8 \mathrm{keVee}$. We use the DAMA modulation amplitudes normalized to $\mathrm{kg}^{-1} \mathrm{day}^{-1} \mathrm{keVee}^{-1}$ in the energy range $1 \mathrm{keVee}<E^{\prime}<8 \mathrm{keVee}$ from Ref.[5]. In both cases we assume a constant quenching factors $q=0.3$ for sodium and $q=0.09$ for iodine, and the energy resolution $\sigma=0.0091\left(\mathrm{E}_{e e} / \mathrm{keVee}\right)+0.448 \sqrt{E_{e e} / \mathrm{keVee}}$ in $\mathrm{keV}$.

The KIMS collaboration operated caesium iodine scintillators with an exposure of $24524.3 \mathrm{~kg}$ days [8]. We obtained the energy resolution extrapolating the two calibration peaks in Fig.2 of [8] at lower energy using the energy dependence $\sigma=\sqrt{a+b E_{e e}}$, while we have used the efficiency from Fig. 1(a) and the measured spectrum and background estimate in the region of interest 2 keVee $<E^{\prime}<4$ keVee from Fig.1(b) of the same paper. We have adopted the quenching factors for both targets from [51]. We have applied background subtraction using the same procedure described for DarkSide-50 and the likelihood of Eq. (B.2).

COSINUS[33] is a next-generation cryogenic scintillating calorimeter using the same targets as DAMA with discrimination between electron and nuclear recoils to suppress the background. We follow the analysis of [52] assuming 5 events with exposure $105 \mathrm{~kg}$ days and an energy 
threshold of $1 \mathrm{keV}$. We have also assumed the energy resolution $\sigma=0.2 \mathrm{keV}$ and taken the nuclear recoil detection efficiency from Eqs. (2.8) and (2.9) of [52].

\section{Appendix B.7. $\mathrm{CaWO}_{4}$ : CRESST-II}

CRESST-II measures heat and scintillation using $\mathrm{CaWO}_{4}$ crystals. We considered the Lise module analysis from [15] with energy resolution $\sigma=0.062 \mathrm{keV}$ and detector efficiency from Fig. 4 of [53]. For our analysis we have selected 15 events for $0.3 \mathrm{keVnr}<E_{R}<0.49 \mathrm{keVnr}$ with an exposure of $52.15 \mathrm{~kg}$ days.

\section{Appendix C. Nuclear response functions for Caesium and Tungsten}

In the case of Caesium in KIMS and of Tungsten in CRESST-II a shell model calculation for the nuclear response functions $W_{l}^{\tau \tau^{\prime}}$ is not available from Refs.[26, 37]. As far as $W_{M}^{\tau \tau^{\prime}}$ is concerned we simply assume a nuclear cross section scaling with the square of the target mass number and a Helm form factor $F_{\text {Helm }}(q r)$ [54] (we take the parametrization of the nuclear radius $r$ from [55]), which, with the normalization conventions of Ref. [26] corresponds for target $T$ to $W_{M}^{\tau \tau^{\prime}}(q)=\left(2 j_{T}+1.\right) /(16 \pi) A_{T}^{2} F_{H e l m}(q r)$. On the other hand, in the case of $W_{\Sigma^{\prime \prime}}^{\tau \tau^{\prime}}$ and $W_{\Sigma^{\prime}}^{\tau \tau^{\prime}}$ we assume $W_{\Sigma^{\prime}}^{\tau \tau^{\prime}}=2 W_{\Sigma^{\prime \prime}}^{\tau \tau^{\prime}}$ and use a Gaussian approximation for the $q^{2}$ dependence. In particular, combining the usual spin-dependent scaling law written as [56]:

$$
S(0)=\frac{1}{\pi} \frac{\left(2 j_{T}+1\right)\left(j_{T}+1\right)}{j_{T}}\left(a_{p}<S_{p}>+a_{n}<S_{n}>\right)^{2}
$$

with the Gaussian form factor [57]:

$$
\frac{S\left(q^{2}\right)}{S(0)}=e^{-q^{2} R^{2} / 4}, \quad R=\left(0.92 A_{T}^{1 / 3}+2.68-0.78 \sqrt{\left(A_{T}^{1 / 3}-3.8\right)^{2}+0.2}\right) \mathrm{fm},
$$

implies:

$$
\begin{aligned}
& W_{\Sigma^{\prime \prime}}^{\tau \tau^{\prime}}\left(q^{2}\right)=\frac{4}{3 \pi} \frac{\left(2 j_{T}+1\right)\left(j_{T}+1\right)}{j_{T}}<S^{\tau}><S^{\tau^{\prime}}>e^{-q^{2} R^{2} / 4} \\
& W_{\Sigma^{\prime}}^{\tau \tau^{\prime}}\left(q^{2}\right)=\frac{8}{3 \pi} \frac{\left(2 j_{T}+1\right)\left(j_{T}+1\right)}{j_{T}}<S^{\tau}><S^{\tau^{\prime}}>e^{-q^{2} R^{2} / 4},
\end{aligned}
$$

with $<S^{0}>=\left(<S_{p}>+<S_{n}>\right) / 2$ and $<S^{1}>=\left(<S_{p}>-<S_{n}>\right) / 2$. For ${ }^{183} \mathrm{~W}$ and ${ }^{333} \mathrm{Cs}$ we take $<S_{p}>=0,<S_{n}>=-0.17$, and $\left\langle S_{p}>=-0.37,<S_{n}>=0.003\right.$, respectively, both from Appendix C of Ref. [58].

\section{Appendix D. The program}

The NRDD_constraints code provides a simple interpolating function written in Python that for a given NR effective coupling calculates the most constraining limit among the experiments listed in Appendix B on the effective WIMP-nucleon cross section $\sigma_{\mathcal{N}}$ defined in Eq.(18) as a function of the WIMP mass $m_{\chi}$ and of the ratio $r=c^{n} / c^{p}$. The code requires the SciPy package and contains only four files, the code NRDD_constraints . py, two data files NRDD_data1.npy and NRDD_data2.npy, and a driver template NRDD_constraints-example.py. The module can be downloaded from 
https://github.com/NRDD-constraints/NRDD

or cloned by

git clone https://github.com/NRDD-constraints/NRDD

By typing:

import NRDD_constraints as NR

two functions are defined. The function sigma_nucleon_bound (inter,mchi, $r$ ) returns the upper bound $\left(\sigma_{\mathcal{N}}\right)_{\text {lim }}$ on the effective cross section of Eq. 18 in $\mathrm{cm}^{2}$ as a function of the WIMP mass mchi and of the ratio $\mathrm{r}=r$ in the ranges $0.1 \mathrm{GeV}<m_{\chi}<1000 \mathrm{GeV},-10^{4}<r<10^{4}$, and contains the same information of Figs. 3416 The inter parameter is a string that selects the interaction term and can be chosen in the list provided by the second function print_interactions():

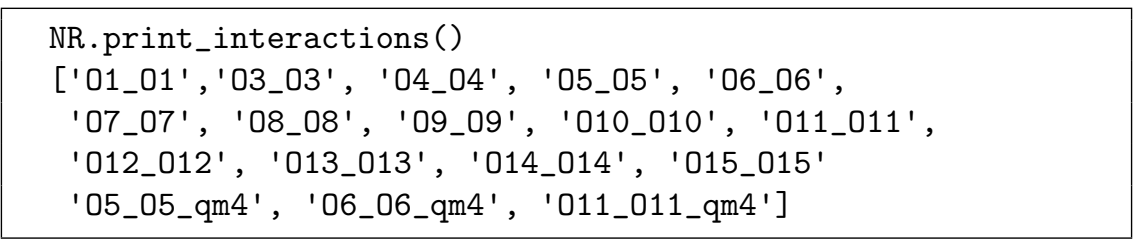

The list above includes also a few examples of long-range interactions where the NR coupling is divided by a propagator term.

\section{References}

[1] P. A. R. Ade, et al., Planck 2013 results. XVI. Cosmological parameters, Astron. Astrophys. 571 (2014) A16. arXiv:1303.5076 doi:10.1051/0004-6361/201321591

[2] R. Bernabei, et al., Searching for WIMPs by the annual modulation signature, Phys. Lett. B424 (1998) $195-201$. doi:10.1016/S0370-2693(98)00172-5

[3] R. Bernabei, et al., First results from DAMA/LIBRA and the combined results with DAMA/NaI, Eur. Phys. J. C56 (2008) 333-355. arXiv:0804.2741 doi:10.1140/epjc/s10052-008-0662-y

[4] R. Bernabei, et al., New results from DAMA/LIBRA, Eur. Phys. J. C67 (2010) 39-49. arXiv:1002.1028 doi: 10.1140/epjc/s10052-010-1303-9

[5] R. Bernabei, et al., First model independent results from DAMA/LIBRA-phase2 arXiv:1805.10486

[6] E. Aprile, et al., Dark Matter Search Results from a One Ton-Year Exposure of XENON1T, Phys. Rev. Lett. 121 (11) (2018) 111302. arXiv: 1805.12562. doi:10.1103/PhysRevLett.121.111302

[7] X. Cui, et al., Dark Matter Results From 54-Ton-Day Exposure of PandaX-II Experiment, Phys. Rev. Lett. 119 (18) (2017) 181302. arXiv:1708.06917 doi:10.1103/PhysRevLett.119.181302

[8] H. S. Lee, et al., Search for Low-Mass Dark Matter with CsI(Tl) Crystal Detectors, Phys. Rev. D90 (5) (2014) 052006. arXiv: 1404.3443 doi:10.1103/PhysRevD.90.052006

[9] R. Agnese, et al., Low-mass dark matter search with CDMSlite, Phys. Rev. D97 (2) (2018) 022002. arXiv: 1707.01632 doi:10.1103/PhysRevD.97.022002

[10] R. Agnese, et al., Results from the Super Cryogenic Dark Matter Search Experiment at Soudan, Phys. Rev. Lett. 120 (6) (2018) 061802. arXiv: 1708.08869 doi:10.1103/PhysRevLett.120.061802

[11] E. Behnke, et al., First Dark Matter Search Results from a 4-kg $\mathrm{CF}_{3} I$ Bubble Chamber Operated in a Deep Underground Site, Phys. Rev. D86 (5) (2012) 052001, [Erratum: Phys. Rev.D90,no.7,079902(2014)]. arXiv: 1204.3094 doi:10.1103/PhysRevD.86.052001,10.1103/PhysRevD.90.079902

[12] E. Behnke, et al., Final Results of the PICASSO Dark Matter Search Experiment, Astropart. Phys. 90 (2017) 85-92. arXiv:1611.01499 doi:10.1016/j.astropartphys.2017.02.005 
[13] C. Amole, et al., Dark Matter Search Results from the PICO-60 CF 3 I Bubble Chamber, Submitted to: Phys. Rev. LarXiv:1510.07754

[14] C. Amole, et al., Dark Matter Search Results from the PICO-60 $\mathrm{C}_{3} \mathrm{~F}_{8}$ Bubble Chamber, Phys. Rev. Lett. 118 (25) (2017) 251301. arXiv:1702.07666 doi:10.1103/PhysRevLett.118.251301

[15] G. Angloher, et al., Results on light dark matter particles with a low-threshold CRESST-II detector, Eur. Phys. J. C76 (1) (2016) 25. arXiv:1509.01515 doi:10.1140/epjc/s10052-016-3877-3

[16] L. T. Yang, et al., Limits on light WIMPs with a $1 \mathrm{~kg}$-scale germanium detector at 160 eVee physics threshold at the China Jinping Underground Laboratory, Chin. Phys. C42 (2) (2018) 023002. arXiv:1710.06650 doi: 10.1088/1674-1137/42/2/023002

[17] A. Aguilar-Arevalo, et al., Search for low-mass WIMPs in a $0.6 \mathrm{~kg}$ day exposure of the DAMIC experiment at SNOLAB, Phys. Rev. D94 (8) (2016) 082006. arXiv: 1607.07410 doi:10.1103/PhysRevD.94.082006

[18] P. Agnes, et al., Low-mass Dark Matter Search with the DarkSide-50 ExperimentarXiv: 1802.06994

[19] D. S. Akerib, et al., First results from the LUX dark matter experiment at the Sanford Underground Research Facility, Phys. Rev. Lett. 112 (2014) 091303. arXiv:1310.8214 doi:10.1103/PhysRevLett.112.091303

[20] D. S. Akerib, et al., Projected WIMP sensitivity of the LUX-ZEPLIN (LZ) dark matter experimentarXiv:1802. 06039

[21] V. Cirigliano, M. L. Graesser, G. Ovanesyan, WIMP-nucleus scattering in chiral effective theory, JHEP 10 (2012) 025. arXiv: 1205.2695 doi:10.1007/JHEP10(2012) 025

[22] V. Cirigliano, M. L. Graesser, G. Ovanesyan, I. M. Shoemaker, Shining LUX on Isospin-Violating Dark Matter Beyond Leading Order, Phys. Lett. B739 (2014) 293-301. arXiv:1311.5886 doi:10.1016/j.physletb. 2014.10.058

[23] B. A. Dobrescu, I. Mocioiu, Spin-dependent macroscopic forces from new particle exchange, JHEP 11 (2006) 005. arXiv:hep-ph/0605342 doi:10.1088/1126-6708/2006/11/005

[24] J. Fan, M. Reece, L.-T. Wang, Non-relativistic effective theory of dark matter direct detection, JCAP 1011 (2010) 042. arXiv: 1008.1591 doi:10.1088/1475-7516/2010/11/042

[25] A. L. Fitzpatrick, W. Haxton, E. Katz, N. Lubbers, Y. Xu, The Effective Field Theory of Dark Matter Direct Detection, JCAP 1302 (2013) 004. arXiv: 1203.3542 doi:10.1088/1475-7516/2013/02/004

[26] N. Anand, A. L. Fitzpatrick, W. C. Haxton, Weakly interacting massive particle-nucleus elastic scattering response, Phys. Rev. C89 (6) (2014) 065501. arXiv: 1308.6288 doi:10.1103/PhysRevC.89.065501

[27] M. Hoferichter, P. Klos, A. Schwenk, Chiral power counting of one- and two-body currents in direct detection of dark matter, Phys. Lett. B746 (2015) 410-416. arXiv: 1503.04811 doi:10.1016/j.physletb.2015.05. 041

[28] R. Catena, P. Gondolo, Global limits and interference patterns in dark matter direct detection, JCAP 1508 (08) (2015) 022. arXiv: 1504.06554 doi:10.1088/1475-7516/2015/08/022

[29] R. Catena, P. Gondolo, Global fits of the dark matter-nucleon effective interactions, JCAP 1409 (09) (2014) 045. arXiv:1405.2637 doi:10.1088/1475-7516/2014/09/045

[30] G. Angloher, et al., Description of CRESST-II datearXiv: 1701.08157

[31] R. Bernabei, et al., The DAMA/LIBRA apparatus, Nucl. Instrum. Meth. A592 (2008) 297-315. arXiv:0804 . 2738 doi:10.1016/j.nima.2008.04.082

[32] E. V. Jáuregui, Talk given at TAUP2017, Sudbury ON, Canada, July 25, 2017, https://indico.cern.ch/event/606690/contributions/2591726/attachments/ 1498457/2332757/Eric_Vazquez_Jauregui_TAUP_2017.pdf

[33] G. Angloher, et al., The COSINUS project - perspectives of a NaI scintillating calorimeter for dark matter search, Eur. Phys. J. C76 (8) (2016) 441. arXiv: 1603.02214 doi:10.1140/epjc/s10052-016-4278-3

[34] K. Schneck, et al., Dark matter effective field theory scattering in direct detection experiments, Phys. Rev. D91 (9) (2015) 092004. arXiv:1503.03379 doi:10.1103/PhysRevD.91.092004

[35] F. Bishara, J. Brod, B. Grinstein, J. Zupan, From quarks to nucleons in dark matter direct detection, JHEP 11 (2017) 059. arXiv:1707.06998 doi:10.1007/JHEP11(2017) 059

[36] S. Kang, S. Scopel, G. Tomar, J.-H. Yoon, Present direct detection sensitivities to WIMP-quark and WIMP-gluon effective interactions arXiv:1810.00607

[37] R. Catena, B. Schwabe, Form factors for dark matter capture by the Sun in effective theories, JCAP 1504 (04) (2015) 042. arXiv: 1501.03729 doi:10.1088/1475-7516/2015/04/042

[38] P. Klos, J. Menndez, D. Gazit, A. Schwenk, Large-scale nuclear structure calculations for spin-dependen WIMP scattering with chiral effective field theory currents, Phys. Rev. D88 (8) (2013) 083516, [Erratum: Phys. Rev.D89,no.2,029901(2014)]. arXiv:1304.7684 doi:10.1103/PhysRevD.89.029901,10.1103/ PhysRevD.88.083516

[39] L. Vietze, P. Klos, J. Menndez, W. C. Haxton, A. Schwenk, Nuclear structure aspects of spin-independent WIMP scattering off xenon, Phys. Rev. D91 (4) (2015) 043520. arXiv:1412.6091 doi:10.1103/PhysRevD.91. 043520 
[40] S. E. Koposov, H.-W. Rix, D. W. Hogg, Constraining the Milky Way potential with a 6-D phase-space map of the GD-1 stellar stream, Astrophys. J. 712 (2010) 260-273. arXiv:0907.1085 doi:10.1088/0004-637X/712/ $1 / 260$

[41] T. Piffl, et al., The RAVE survey: the Galactic escape speed and the mass of the Milky Way, Astron. Astrophys. 562 (2014) A91. arXiv:1309.4293 doi:10.1051/0004-6361/201322531

[42] P. Salucci, F. Nesti, G. Gentile, C. F. Martins, The dark matter density at the Sun's location, Astron. Astrophys. 523 (2010) A83. arXiv: 1003.3101 doi:10.1051/0004-6361/201014385

[43] F. Nesti, P. Salucci, The Dark Matter halo of the Milky Way, AD 2013, JCAP 1307 (2013) 016. arXiv: 1304.5127 doi:10.1088/1475-7516/2013/07/016

[44] S. Kang, S. Scopel, G. Tomar, J.-H. Yoon, DAMA/LIBRA-phase2 in WIMP effective models, JCAP 1807 (07) (2018) 016. arXiv: 1804.07528 doi:10.1088/1475-7516/2018/07/016

[45] R. Catena, A. Ibarra, S. Wild, DAMA confronts null searches in the effective theory of dark matter-nucleon interactions, JCAP 1605 (05) (2016) 039. arXiv: 1602.04074 doi:10.1088/1475-7516/2016/05/039

[46] S. Yellin, Finding an upper limit in the presence of an unknown background Phys. Rev. D 66 (2002) 032005 doi:10.1103/PhysRevD.66.032005 URL https://link.aps.org/doi/10.1103/PhysRevD.66.032005

[47] E. Aprile, et al., Signal Yields of keV Electronic Recoils and Their Discrimination from Nuclear Recoils in Liquid Xenon, Phys. Rev. D97 (9) (2018) 092007. arXiv:1709.10149 doi:10.1103/PhysRevD.97.092007

[48] Z. Ahmed, et al., Analysis of the low-energy electron-recoil spectrum of the CDMS experiment, Phys. Rev. D81 (2010) 042002. arXiv:0907.1438 doi:10.1103/PhysRevD.81.042002

[49] J. Lindhard, V. Nielsen, M. Scharff, P. V. Thomsen, Integral Equations Governing Radiation Effects (Notes On Atomic Collisions III ) Mat. Fys. Medd. Dan. Vid. Selsk. 33. URL/http://www.sdu.dk/media/bibpdf/Bind30-39/Bind/mfm-33-10.pdf

[50] C. Amole, et al., Dark Matter Search Results from the PICO-2L C ${ }_{3} \mathrm{~F}_{8}$ Bubble Chamber, Phys. Rev. Lett. 114 (23) (2015) 231302. arXiv:1503.00008 doi:10.1103/PhysRevLett.114.231302

[51] H. Park, D. Choi, J. Choi, I. Hahn, M. Hwang, W. Kang, H. Kim, J. Kim, S. Kim, S. Kim, T. Kim, Y. Kim, Y. Kwon, H. Lee, J. Lee, M. Lee, S. Lee, S. Noh, I. Park, E. Seo, E. Won, H. Yang, M. Yang, I. Yu, Neutron beam test of csi crystal for dark matter search Nuclear Instruments and Methods in Physics Research Section A: Accelerators, Spectrometers, Detectors and Associated Equipment 491 (3) (2002) 460 - 469. doi : https : //doi.org/10.1016/S0168-9002(02)01274-3

URL http://www.sciencedirect.com/science/article/pii/S0168900202012743

[52] F. Kahlhoefer, F. Reindl, K. Schffner, K. Schmidt-Hoberg, S. Wild, Model-independent comparison of annual modulation and total rate with direct detection experiments $\operatorname{arXiv:1802.10175}$

[53] G. Angloher, et al., Description of CRESST-II datiarXiv: 1701.08157

[54] R. H. Helm, Inelastic and Elastic Scattering of 187-Mev Electrons from Selected Even-Even Nuclei, Phys. Rev. 104 (1956) 1466-1475. doi:10.1103/PhysRev.104.1466

[55] G. Duda, A. Kemper, P. Gondolo, Model Independent Form Factors for Spin Independent Neutralino-Nucleon Scattering from Elastic Electron Scattering Data, JCAP 0704 (2007) 012. arXiv:hep-ph/0608035 doi: 10. 1088/1475-7516/2007/04/012

[56] J. Engel, S. Pittel, P. Vogel, Nuclear physics of dark matter detection, Int. J. Mod. Phys. E1 (1992) 1-37. doi: 10.1142/S0218301392000023

[57] G. Belanger, F. Boudjema, A. Pukhov, A. Semenov, Dark matter direct detection rate in a generic model with micrOMEGAs 2.2, Comput. Phys. Commun. 180 (2009) 747-767. arXiv:0803.2360 doi:10.1016/j.cpc. 2008.11.019

[58] V. A. Bednyakov, F. Simkovic, Nuclear spin structure in dark matter search: The Zero momentum transfer limit, Phys. Part. Nucl. 36 (2005) 131-152, [Fiz. Elem. Chast. Atom. Yadra36,257(2005)]. arXiv: hep-ph/0406218 